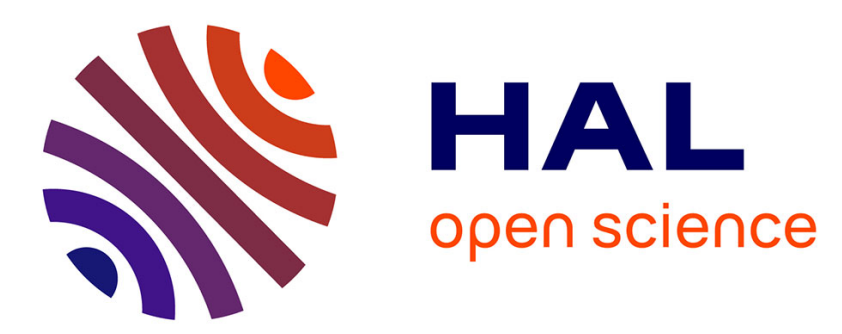

\title{
Effet Mössbauer et techniques complémentaires : III. - L'EXAFS appliqué aux déterminations structurales de milieux désordonnés
}

D. Raoux, J. Petiau, P. Bondot, G. Calas, A. Fontaine, P. Lagarde, P. Levitz, G. Loupias, A. Sadoc

\section{To cite this version:}

D. Raoux, J. Petiau, P. Bondot, G. Calas, A. Fontaine, et al.. Effet Mössbauer et techniques complémentaires: III. - L'EXAFS appliqué aux déterminations structurales de milieux désordonnés. Revue de Physique Appliquée, 1980, 15 (6), pp.1079-1094. 10.1051/rphysap:019800015060107900 . jpa00244826

\section{HAL Id: jpa-00244826 https://hal.science/jpa-00244826}

Submitted on 1 Jan 1980

HAL is a multi-disciplinary open access archive for the deposit and dissemination of scientific research documents, whether they are published or not. The documents may come from teaching and research institutions in France or abroad, or from public or private research centers.
L'archive ouverte pluridisciplinaire HAL, est destinée au dépôt et à la diffusion de documents scientifiques de niveau recherche, publiés ou non, émanant des établissements d'enseignement et de recherche français ou étrangers, des laboratoires publics ou privés. 


\title{
Effet Mössbauer et techniques complémentaires :
}

\section{III. - L'EXAFS appliqué aux déterminations structurales de milieux désordonnés}

\author{
D. Raoux $(*)$, J. Petiau $\left({ }^{* *}\right)\left({ }^{* *}\right)$, P. Bondot $\left({ }^{* *}\right)$, G. Calas $\left({ }^{* *}\right)$, A. Fontaine $\left(^{*}\right)$, \\ P. Lagarde $(*)$, P. Levitz $\left({ }^{* *}\right)$, G. Loupias $\left({ }^{* *}\right)$ et A. Sadoc $\left({ }^{* * * *}\right)$
}

Laboratoire pour l'Utilisation du Rayonnement Electromagnétique (Laboratoire du C.N.R.S. associé à l'Université Paris-Sud), Bât. 209C, Université Paris-Sud, 91405 Orsay, France

(Reçu le 14 novembre 1979, révisé le 17 janvier 1980, accepté le 29 janvier 1980)

Résumé. - Depuis quelques années, et avec l'avènement du rayonnement synchrotron, l'EXAFS devient une technique de plus en plus utilisée pour l'étude de l'ordre local dans les systèmes désordonnés ou très dilués. C'est en effet, dans son principe, une expérience très simple qui consiste à mesurer l'absorption des photons $\mathrm{X}$ par la matière, et qu'on réalise facilement lorsqu'on dispose de hauts flux de photons émis par les anneaux de stockage d'électrons ou de positrons. Elle a aussi des spécificités uniques par rapport aux techniques de diffusion des rayons $\mathrm{X}$ ou des neutrons :

- elle n'est sensible qu'à l'ordre local et ne sonde qu'une ou deux couches atomiques autour d'un atome donné ;

- en contrepartie, elle est très précise et donne les distances à $1 / 100 \AA ̊$ près ;

- elle est très sensible : on peut étudier des systèmes dilués avec des concentrations de $10^{-4}$;

- dans un système complexe, l'EXAFS peut donner l'ordre local autour de chacun des constituants.

Cependant, l'analyse d'un spectre EXAFS n'est pas aussi directe que ne l'est l'interprétation d'un spectre de diffusion des rayons $\mathrm{X}$ ou des neutrons. La théorie de l'EXAFS implique diverses approximations que l'on ne doit pas perdre de vue sous peine d'aboutir à des résultats erronés. Un point crucial est l'extrême sensibilité de l'EXAFS au désordre de position qui peut en limiter l'emploi pour l'étude des milieux désordonnés.

Ces différents points sont discutés dans la première partie de cet article, où on décrit aussi les montages expérimentaux dont on dispose à LURE. La seconde partie présente un certain nombre d'exemples d'utilisation de l'EXAFS pour l'étude des structures locales dans les amorphes, mais aussi les liquides, et plus généralement les systèmes désordonnés (cœurs de défauts, composés non stœchiométriques, etc...).

Abstract. - Since a few years, EXAFS has become popular as a new tool for structural studies of local order in disordered systems, as well as in dilute ones. The first reason for such a boom is that it is a very simple absorption measurement experiment, which is easily performed as soon as one gets the high photon fluxes emitted in the Xray range by the synchrotrons and electron storage rings. It has also unique capabilities in comparison to the scattering of X-rays or neutrons :

- it is a very local probe which is limited to the study of the first or second coordination shell around a given atom;

— distances may be measured with a $0.01 \AA$ accuracy ;

- it is very sensitive : dilute samples with concentrations in the range of $10^{-4}$ can be studied ;

- in a complex system, it gives the local structure around each constituant.

However, the analysis of the spectra is not so direct as that of a scattering experiment, and the theory on which it is based, implies some approximations which one has to keep in mind in order to derive correct findings. A crucial point is the large sensitivity of EXAFS to disorder which may give a serious limitation in its use as a conventional technique.

These points are discussed in part I of this paper, which also presents the experimental set-up we have at LURE. In part II, various examples are given which concern the local structure of amorphous compounds, as well as that of liquids or other kinds of disordered systems (cores of defects, non stoichiometric compounds, etc...).

$\left(^{*}\right)$ Laboratoire de Physique des Solides (LA 02), Université Paris-Sud, Bât. 510, Orsay, France.

(**) Laboratoire de Minéralogie-Cristallographie (LA 09), Université P.-et-M.-Curie, Paris, France.
$(* * *)$ E.N.S.J.F., Paris.

$\left({ }^{* * * *}\right)$ Laboratoire de Physique Cristalline (ERA 13), Université Paris-Sud, Orsay, France. 
Par effet photoélectrique, un photon peut arracher un électron à un atome pourvu que son énergie $\hbar \omega$ soit supérieure à l'énergie de liaison $E_{\mathrm{L}}$ de l'électron. Pour des photons $\mathrm{X}$, on observe donc une série de discontinuités dans la variation de l'absorption en fonction de l'énergie, les seuils $K$, $L$, qui correspondent à des excitations d'électrons $1 \mathrm{~s}, 2 \mathrm{~s}$ et $2 \mathrm{p}$ vers des états du continuum (Fig. 1).

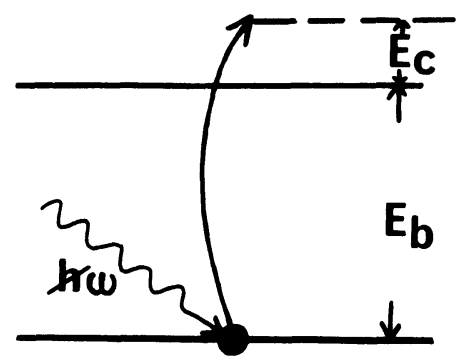

Fig. 1. - Absorption d'un photon d'énergie $\hbar \omega$ par un atome : un électron d'une couche profonde d'énergie $E_{\mathrm{b}}$ est éjecté avec une énergie cinétique : $E_{\mathrm{c}}=\hbar \omega-E_{\mathrm{b}}$.

[Absorption of a X-ray photon in the innershell of an atom. A photoelectron is emitted with a kinetic energy : $E_{\mathrm{c}}=\hbar \omega-E_{\mathrm{b}}$ where $E_{\mathrm{b}}$ is the binding energy of the core electron and $\hbar \omega$ the energy of the impinging photon.]

Au-delà du seuil l'absorption décroît sur quelques centaines d'électrons-volts, comme le montre la figure 2 qui compare les spectres d'absorption $\mathrm{K}$ du néon à l'état gazeux et à l'état solide $\left(E_{\mathrm{K}}=867,1 \mathrm{eV}\right)$ [1]. $\mathrm{Au}$ voisinage du seuil, les deux spectres sont très semblables et présentent des pics étroits, de largeurs inférieures à la résolution expérimentale $0,3 \mathrm{eV}$, qui ne dépendent pas de la température. Ces structures, souvent dites de Kossel [2], sont d'origine atomique puisqu'on les observe dans une vapeur de gaz néon monoatomique à basse pression (1 torr), aussi bien

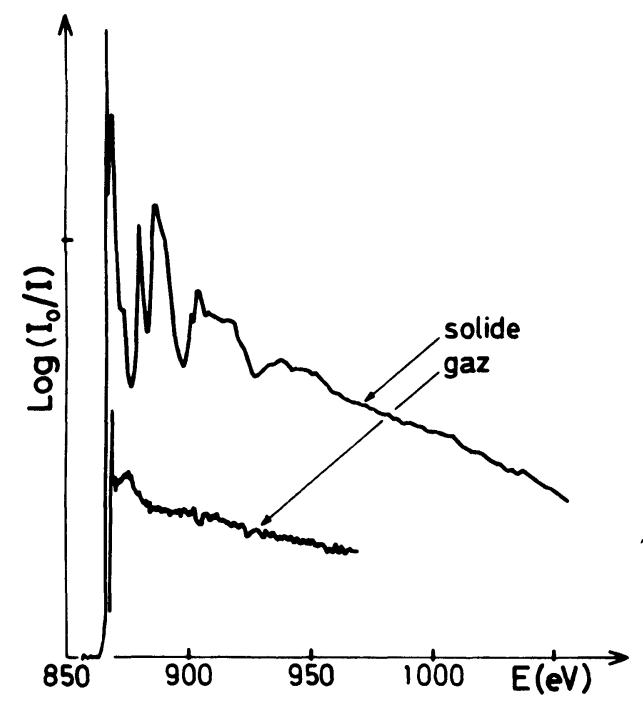

Fig. 2. - Absorption en fonction de l'énergie des photons $\mathrm{X}$ incidents pour le seuil $\mathrm{K}$ du néon, à l'état solide et gazeux.

[K-edge absorption spectrum of neon in the vapour and solid state. The energy scale is that of the impinging photons.] que dans le solide. Elles correspondent dans le cas présent à des transitions entre l'état initial $1 \mathrm{~s}$ de l'électron et des états finaux liés $3 p$ et $4 p$. Leur largeur résulte d'une convolution des largeurs des états a tomiques initial et final. Au-delà de 30 à $50 \mathrm{eV}$ du seuil les deux spectres sont par contre différents : dans la phase vapeur, l'absorption décroît de façon monotone, tandis qu'elle est modulée dans la phase solide par les oscillations de Kronig [3] bien connues dès les années 30, mais qui ont été rebaptisées vers 1970 du nom d'EXAFS, abréviation d'Extended X-Ray Absorption Fine Structure.

Ces oscillations ont des périodes qui croissent avec l'énergie $E=\hbar \omega-E_{\mathrm{b}}$ du photoélectron et qui sont très grandes devant les largeurs des structures au seuil. Typiquement leurs périodes sont de 50 à $100 \mathrm{eV}$ en énergie, et elles dépendent de l'entourage de l'atome photoexcité. Elles peuvent s'étendre jusqu'à 800 ou $1000 \mathrm{eV}$ du seuil, et décroissent avec la température. Dès les années 30, elles ont été interprétées comme résultant de la rétrodiffusion par les atomes voisins du photoélectron éjecté de l'atome central $[3,4,5]$. Ces théories initiales considéraient des modulations de la densité d'états finaux dues à des interférences entre l'onde électronique primaire sortant de l'atome central photoexcité et les ondes diffractés par le cristal (cf. Fig. 3); l'absorption est en

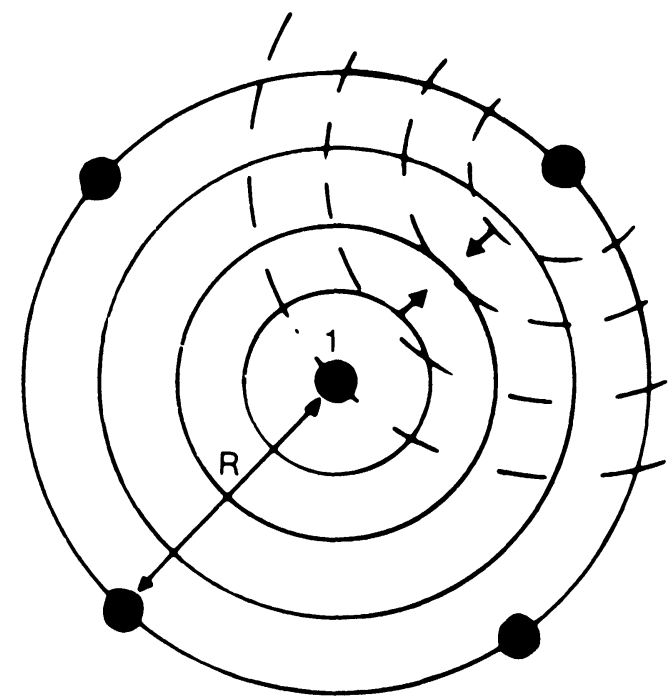

Fig. 3. - Le photoélectron éjecté de l'atome central se propage sous forme d'une onde sphérique $(-)$ et est rétrodiffusé par les atomes voisins $(--\longrightarrow)$

[The photoelectron is emitted as a spherical wave (-) and is backscattered towards the central atom by the neighbouring atoms $(---)$.]

effet donnée par la règle d'or de Fermi qui, dans l'approximation dipolaire $\left({ }^{1}\right)$, s'écrit :

( $\left.{ }^{1}\right)$ Cette approximation est bien vérifiée : prenons le cas du seuil $\mathrm{K}$ de l'arsenic. La longueur d'onde $\mathrm{X}$ qui lui est associée est voisine de $1 \AA$, tandis que le diamètre de l'orbite 1 s de As est de l'ordre de $3 \times 10^{-2} \AA$. 


$$
\mu \sim \sum_{\mathrm{f}}|\langle\mathrm{i}|\varepsilon . \mathbf{r}| \mathrm{f}\rangle|^{2} \delta\left(E_{\mathrm{f}}-E_{\mathrm{i}}-\hbar \omega\right)
$$

où $|\mathrm{i}\rangle$ est l'état initial de l'électron (par exemple 1s pour un seuil $\mathrm{K}$ ),

|f $>$ l'état final de symétrie donnée ( $p$ pour un seuil $\mathrm{K})$,

$\varepsilon$ est le vecteur polarisation du champ électrique.

Cette formule fait intervenir la densité d'états partielle dans l'état final ( $p$ pour un seuil $\mathrm{K}$ ) mais aussi un élément de matrice qui dépend de la forme de l'état final $|\mathrm{f}\rangle$, et par là de la rétrodiffusion de l'onde électronique par les atomes voisins; c'est en fait les modulations de cet élément de matrice qui sont responsables des modulations EXAFS, ce qui explique le peu de succès quantitatif des premières théories.

Ce peu de succès, joint à la difficulté de mesurer un spectre d'absorption s'étendant sur un millier d'électrons-volts avec le fond continu émis par un tube à rayons $\mathrm{X}$, explique que les oscillations de Kronig aient peu été utilisées à des études structurales jusqu'au début des années 70. C'est le mérite de Stern, Lytle et Sayers [6-10] d'avoir montré qu'elles pouvaient être utilisées comme une sonde pour l'ordre local permettant des mesures précises, à 1 ou 2 centièmes d'Å près, des distances entre proches voisins. Leur interprétation, qui est dans ses grandes lignes celle utilisée maintenant et est développée au paragraphe 1.3, repose sur la faible valeur du libre parcours moyen d'un électron dans la matière condensée qui pour des énergies supérieures à une cinquantaine d'eV n'est que de quelques $\AA$, typiquement $5 \AA$ (Fig. 4).

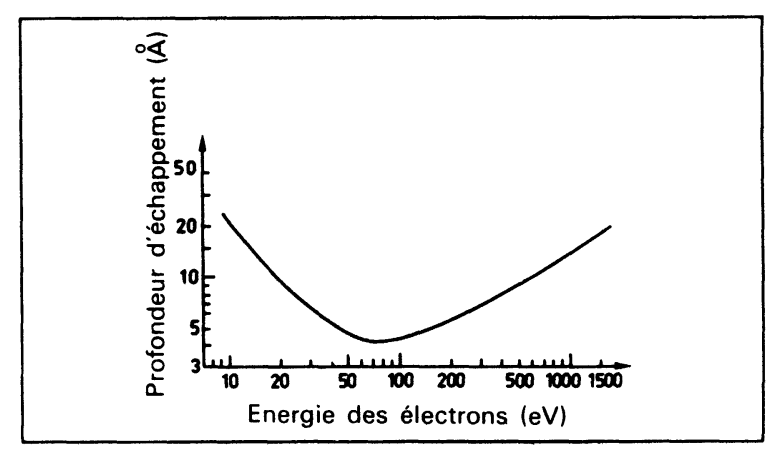

Fig. 4. - Couche semi-universelle donnant le libre parcours moyen des électrons en fonction de leur énergie.

[Semi-universal curve showing the dependence of the electron mean free path in a solid on its kinetic energy.]

L'EXAFS est de ce fait une sonde très locale; son interprétation est simple dans la mesure où on peut négliger le plus souvent les phénomènes de diffusion multiples qui rendent difficile l'analyse des expériences de diffraction d'électrons lents DEL, par exemple. La précision obtenue sur la mesure des distances entre proches voisins explique en partie le très rapide développement de cette technique depuis 1974.

L'autre raison de ce boom est le remplacement du rayonnement $\mathrm{X}$ émis par les tubes conventionnels par le rayonnement émis par les anneaux de stockage à électrons ou positrons comme Spear à Stanford, Do ris à Hambourg et $\mathrm{ACO}$ et $\mathrm{DCI}$ à Orsay. Le gain d'intensité par rapport au fond continu d'un tube conventionnel est de $10^{4}$ à $10^{5}$ [11]. On fait donc à DCI une mesure en $20 \mathrm{~min}$. avec un signal sur bruit supérieur à celui obtenu après un mois d'exposition sur un tube. Cette amélioration du rapport signal sur bruit permet de mesurer l'EXAFS à de hautes énergies du photoélectron - disons $E_{\mathrm{c}}>500 \mathrm{eV}$ pour lesquelles la théorie simple exposée plus loin est bien établie. Il en a résulté un développement très rapide des théories quantitatives de l'EXAFS [1214] qui ont été testées avec succès ces 4 dernières années par les équipes d'expérimentateurs travaillant dans les centres de rayonnement synchrotron. Les possibilités d'application de l'EXAFS sont déjà très variées et vont de la biologie à la physique des surfaces en passant par la chimie, la physique des solides, des liquides, la physique des défauts, ou même la géologie! (voir l'article général d'Eisenberger et Kincaid [15]).

Avant de résumer dans une première partie l'état actuel de la compréhension quantitative de l'EXAFS et de décrire comment on fait une expérience et comment on l'analyse, puis de donner dans une deuxième partie quelques exemples d'application à la physique des milieux mal ordonnés, il est peut-être utile de préciser dès cette introduction les spécificités avantageuses ou désavantageuses de l'EXAFS par rapport aux autres techniques de mesure de l'ordre local comme la diffusion des rayons $\mathrm{X}$ ou des neutrons.

1) $\mathrm{Du}$ fait du libre parcours moyen de l'électron, l'EXAFS est une sonde très locale qui souvent ne voit guère au-delà des premiers et seconds voisins. Inversement, la précision des mesures de distance est souvent de l'ordre de $1 \times 10^{-2} \AA$ pour la distance entre proches voisins (alors qu'elle est plutôt de l'ordre de $5 \times 10^{-2}$ à $0,1 \AA$ en diffusion des rayons X).

2) L'EXAFS est une méthode très sélective puisqu'on excite séparément les seuils $\mathrm{K}$ ou $\mathrm{L}$ des différents éléments (ils sont séparés de plusieurs centaines d'électrons-volts). Elle permet donc d'étudier des systèmes très dilués $\left(C \sim\right.$ quelque $10^{-3}$ par une mesure d'absorption, $C \sim$ quelque $10^{-5}$ en mesurant l'intensité de la fluorescence $X$, cf. $\S 1.1)$. Cette sensibilité a été beaucoup employée pour des études de biophysique et plus récemment pour des études de défauts (impuretés).

3) La sélectivité permet aussi de séparer directement certaines fonctions de distribution de paires : dans le cas d'un mélange binaire $A B$, par exemple, les paires B-B ne contribuent pas à l'EXAFS sur le seuil de l'élément $A$, et réciproquement. D'où son intérêt pour l'étude de systèmes complexes (elle peut remplacer les techniques de substitution isotopique employée en diffusion des neutrons). 
4) L'EXAFS n'a absolument pas besoin de l'invariance de translation du cristal : on peut l'appliquer à tout système ordonné, ou mal ordonné à grande ou moyenne distance pourvu qu'il existe un ordre local radial. D'où l'application à l'étude des amorphes ou des liquides.

5) L'EXAFS est très sensible au degré d'ordre radial : de faibles fluctuations, statiques ou dynamiques, des distances entre premiers voisins amortissent très fortement le signal en fonction de l'énergie $E_{\mathrm{c}}$ du photoélectron. D'où une potentialité d'emploi pour des mesures fines d'ordre local - qui n'a pas encore été beaucoup utilisée - mais aussi une limitation d'emploi à l'étude de systèmes pas trop désordonnés à l'échelle locale. Ce point important pour la physique des liquides et amorphes est discuté plus loin.

1. 1 LES DISPOSITIFS EXPÉRIMENTAUX. - La figure 5 donne le schéma de principe d'un montage de spectroscopie d'absorption. Le faisceau $\mathrm{X}$ blanc émis par l'anneau de stockage est collimaté par une fente d'entrée qui le délimite. Typiquement, le faisceau utile a $1 \mathrm{~cm}^{2}$ de section pour le montage d'X mous d'ACO (550-3 $000 \mathrm{eV})$, et $2 \mathrm{~mm}$ de hauteur sur $4 \mathrm{~cm}$ de large pour le montage en $\mathrm{X}$ conventionnels de DCI $(3500-20000 \mathrm{eV})$.

Le monochromateur est un système de deux cristaux parallèles à ACO [16] ou un monocristal dans lequel on a taillé deux faces parallèles à DCI. Le faisceau X subit donc deux réflexions de Bragg et ressort parallèlement à la direction incidente. La résolution est typiquement de l'ordre de $2 \times 10^{-4}$ pour les monochromateurs à silicium ou germanium employés à DCI. Sur ACO elle dépend beaucoup du cristal utilisé et varie de 0,3 à 3 ou $4 \mathrm{eV}$ (béryl, mica, gypse, KAP...). Du fait de la très forte absorption des $\mathrm{X}$ mous par la matière, l'expérience sur ACO est sous un bon vide $\left(10^{-7}\right.$ torr), avec toutes les servitudes que cela implique.
On mesure l'absorption optique à travers l'échantillon en mesurant par des chambres à ionisation l'intensité du faisceau $I_{0}$ avant et $I$ après l'échantillon (montage sur DCI). Pour le montage d'ACO, on mesure successivement $I_{0}$ et $I$ avec divers détecteurs (channeltron ou galette de microcanaux dans le domaine $500-1500 \mathrm{eV}$, chambre à ionisation au-delà de $1500 \mathrm{eV}$ ) [17].

Les deux expériences sont pilotées par des ordinateurs qui assurent la rotation du monochromateur, et le stockage des intensités $I$ et $I_{0}$. Typiquement la durée d'acquisition d'un spectre EXAFS de 1000 points est de 20 à 30 min sur DCI, de 2 à $3 \mathrm{~h}$ sur ACO. Les échantillons peuvent être des monocristaux, des poudres, des liquides : le problème est l'épaisseur qui, pour le montage en $\mathrm{X}$ conventionnels de DCI est typiquement $20 \mu$, et en X mous sur ACO de $5000 \AA$ à 1 ou $2 \mu$, ce qui peut limiter l'utilisation du spectromètre $X$ mous dans certains cas.

Les deux montages permettent d'accéder à pratiquement tous les éléments à partir du fluor, soit en enregistrant les seuils $\mathrm{K}$, soit les seuils $\mathrm{L}$ (avec des trous pour le phosphore et le chlore). Le montage sur DCI est assez classique et couvre le même domaine d'énergies que ceux de Stanford ou Hambourg, tandis que le montage en $\mathrm{X}$ mous est à l'heure actuelle unique.

Il existe d'autres manières de mesurer l'absorption : on peut mesurer l'intensité de la fluorescence $\mathrm{X}$ avec un détecteur solide qui permet de l'isoler du fond dû à la diffusion Rayleigh ou Compton. Elle est proportionnelle à l'absorption. L'intérêt d'une telle méthode est que l'absorption due aux atomes autres que celui qui est photoexcité ne donne pas de fluorescence : on efface ainsi les autres atomes, d'où une augmentation de la sensibilité, au prix d'un temps de comptage beaucoup plus grand. Cette méthode a été employée avec succès à Stanford notamment pour des études de biophysique $[18,19]$ et permet de travailler avec de faibles concentrations, de l'ordre de quelque $10^{-5}$. Un dispositif de ce type est maintenant disponible

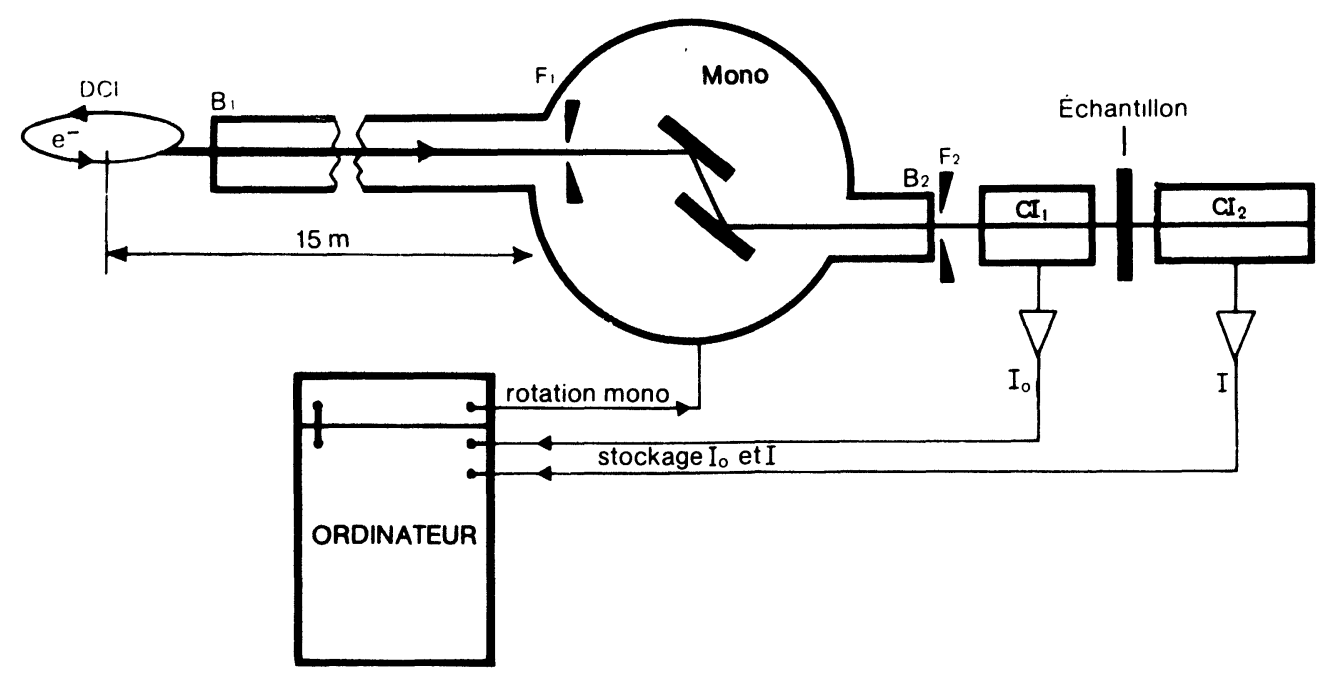

Fig. 5. - Schéma de principe d'une mesure d'EXAFS par absorption.

[Schematic picture of a X-ray absorption experiment.] 
sur le montage de DCI. On peut aussi mesurer l'intensité des électrons Auger réémis.

Les énergies des Auger sont spécifiques d'un atome donné, comme la fluorescence $\mathrm{X}$; on peut donc ainsi détecter également des espèces très diluées. Cette technique a permis de mesurer l'EXAFS dû à une monocouche d'iode sur une surface d'argent (111), avec un temps de mesure de l'ordre de $5 \mathrm{~h}$ à Stanford [20]. La détection de l'EXAFS par les photoélectrons secondaires réémis par la relaxation de l'atome photoexcité a été utilisée dans le domaine des $\mathrm{X}$ très mous ( 200 à $500 \mathrm{eV}$ ) par différentes équipes de Stanford [21, 22]. Du fait du faible libre parcours moyen électronique, cette technique permet de mesurer l'EXAFS dû à la surface de l'échantillon, en sélectionnant des énergies d'électrons pour lesquelles le libre parcours est de l'ordre de quelques $\AA$. Cette technique très prometteuse pour la physique des surfaces présente en principe l'avantage de la simplicité d'analyse de l'EXAFS par rapport aux expériences de diffraction des électrons lents. Un montage de ce type est en préparation sur l'anneau d'ACO.

1.2 LES SEUILS D'ABSORPTION COMME SONDE DE CARACTÉRISATION PHYSICO-CHIMIQUE. - La mesure - avec une bonne résolution en énergie - de l'absorption au seuil, qui fait partie de la mesure globale d'EXAFS, peut fournir simplement des renseignements qualitatifs, ou même quantitatifs, sur l'état physico-chimique de l'atome excité ou sur son entourage, et ce, soit par la mesure du déplacement chimique du seuil, soit par l'étude des raies blanches, ou plus généralement de la forme du seuil d'absorption.

Un exemple de raies blanches a été donné dans la figure 2 relative au néon dans l'état gazeux ou solide. Elles peuvent être décrites dans le langage de la physique atomique comme des raies de Rydberg, ou dans le langage de la physique des solides comme des excitations atomiques : il s'agit des transitions du photoélectron vers des états liés au trou en couche profonde créé par l'effet photoélectrique [23]. Ces structures peuvent résulter de mécanismes différents, ce qui en rend l'interprétation délicate : transition vers des états moléculaires [24] ou solides [25] vides et de symétrie convenable, résonances correspondant à des niveaux non liés situés au-dessus du seuil d'ionisation [26], sans parler des effets à $N$ corps dans l'état final [27] qui ont fait l'objet de nombreuses discussions. Il existe depuis les années 30 une abondante littérature sur l'interprétation des seuils d'absorption, notamment dans les métaux, en liaison avec les résultats d'émission $\mathrm{X}$, de photoémission (XPS) qui dépasse largement l'objet de cet article. Malgré les difficultés d'interprétation, ce domaine est très prometteur, notamment du fait de la facilité avec laquelle ces mesures d'absorption au seuil peuvent être faites (à DCI une telle mesure ne dure que quelques minutes). L'observation de ces raies fournit en effet des renseignements directs sur les densités d'états partielles vides situés immédiatement au-dessus du niveau de Fermi [28, 29]. Elle peut également fournir des informations sur la structure au voisinage de l'atome photoexcité, dans la mesure où la force d'oscillateur associée à une telle raie dépend de la symétrie locale.

Lytle [30], par exemple, a montré que dans $\mathrm{CrVO}_{4}$ c'est le vanadium qui forme un radical $\mathrm{VO}_{4}$ plutôt que le chrome, information que ne pouvait donner la diffusion des rayons $\mathrm{X}$ du fait de la similitude des facteurs de diffusion atomique pour $\mathrm{V}$ et $\mathrm{Cr}$.

Probablement beaucoup plus faciles à interpréter que les formes des seuils, les déplacements chimiques des seuils d'absorption $\mathbf{X}$ permettent une estimation directe de l'état d'ionisation de l'atome photoexcité ; comme on peut le voir sur la figure 6, l'énergie

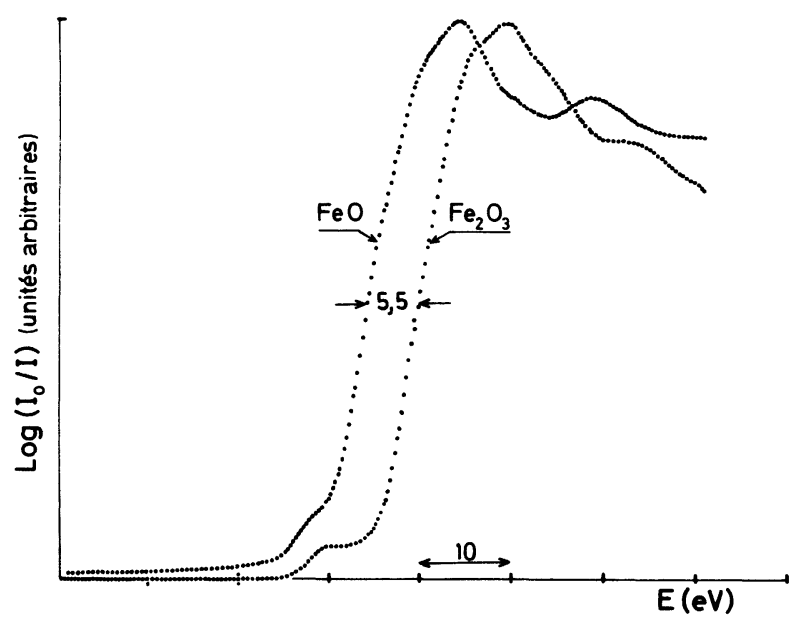

Fig. 6. - Déplacement du seuil d'absorption $\mathrm{K}$ du fer en fonction de l'état d'oxydation.

[Shift of the $\mathrm{K}$ edge of iron with the oxidation state.]

du seuil $\mathrm{K}$ du fer croît avec le degré d'ionisation. En comparant donc les seuils dans une situation inconnue à des étalons simples, on peut déterminer l'état d'ionisation sans avoir à rendre compte théoriquement des amplitudes de déplacement chimique. Cette méthode est employée à LURE pour caractériser l'état chimique du fer en impuretés dans des verres sodés [31]. Elle permet même de déterminer des valences intermédiaires. A la suite des travaux de H. Launois et al. [32], plusieurs équipes utilisent cette technique à LURE pour déterminer la valence de terres rares dans le cas où elle peut prendre deux valeurs (fluctuations de valence). On peut même ainsi apprécier les proportions relatives d'un mélange de valence à quelque $10 \%$ près, avec une sensibilité tout à fait comparable à celle obtenue par les mesures de susceptibilité magnétique.

1.3 Formulation SIMPLE de L'EXAFS. - La probabilité d'absorption, donnée par la formule (1) ci-dessus, est évidemment valable quel que soit l'état final $|\mathrm{f}\rangle$. Pour un atome isolé, comme dans une 
vapeur monoatomique, l'état final électronique est, à l'intérieur de l'atome, un état atomique $\left|\mathrm{f}_{0}\right\rangle$ et à l'extérieur une onde sphérique, de symétrie $p$ pour une absorption en couche $\mathrm{K}\left({ }^{2}\right)$, sortant de l'atome et qu'on peut donc décrire par une fonction d'onde :

$$
Y_{1,0}\left(\frac{\mathbf{r}}{r}\right) h_{1}^{(1)}(k r) \mathrm{e}^{\mathrm{i} \delta_{1}^{\prime}(k)}
$$

où $Y_{1,0}$ est l'harmonique sphérique $l=1, m=0$ polarisée parallèlement au champ électrique, $h_{1}^{(1)}(k r)$ la fonction de Hankel du premier type pour $l=1$ avec $k=$ moment de l'électron. Le déphasage $\delta_{1}^{\prime}(k)$ permet d'écrire le raccordement entre l'onde électronique sphérique à l'extérieur de l'atome, supposée se propager dans le vide, et l'état électronique $\left|f_{0}\right\rangle$ de l'atome photoexcité, c'est-à-dire avec un trou électronique dans la couche $1 \mathrm{~s}$.

Comme l'état initial $\mid$ i $\rangle$, par exemple un état 1s, est très localisé, l'élément de matrice qui figure dans (1) peut être calculé au noyau de l'atome, et on obtient pour un atome isolé

$$
\mu_{0} \sim\left|\left\langle\mathrm{i}|r| \mathrm{f}_{0}\right\rangle\right|^{2} \delta\left(E_{\mathrm{f}_{0}}-E_{\mathrm{i}}-\hbar \omega\right) .
$$

La présence d'un atome en $r_{1}$ modifie l'état final en donnant naissance à l'EXAFS. On peut visualiser cet effet de façon simple.

L'onde sortant de l'atome photoexcité est assimilée, au voisinage de l'atome diffuseur situé en $\mathbf{r}_{1}$, à une onde plane : sa fonction d'onde est donnée par la limite asymptotique de la fonction de Hankel $h_{1}^{(1)}(k r)$ soit

$$
\frac{i \mathrm{e}^{i\left(k r_{1}+\delta_{1}^{\prime}\right)}}{2 k r_{1}} .
$$

Elle est rétrodiffusée par l'atome diffuseur avec une amplitude de diffusion $f(\pi, k)$ qui est complexe et donnée par la formule classique

$$
f(\pi, k)=\frac{1}{2 i k} \sum_{l}(2 l+1)\left(\mathrm{e}^{2 i \delta_{l}}-1\right) P_{l}(-1) .
$$

L'onde rétrodiffusée se propage sous forme d'une onde sphérique qui revient sur l'atome central avec une amplitude proportionnelle à

$$
\frac{\mathrm{e}^{i k r_{1}}}{r_{1}} \mathrm{e}^{i \delta_{1}^{\prime}(k)}
$$

puisqu'elle subit à nouveau à son retour sur l'atome central le déphasage créé par le potentiel dans l'atome central.

La fonction d'onde dans l'état final, à l'intérieur de l'atome central s'écrit donc

$$
|\mathrm{f}\rangle=\left|\mathrm{f}_{0}\right\rangle\left\{1+\frac{i f(\pi, k)}{2 k r_{1}^{2}} \mathrm{e}^{2 i\left(\delta_{1}^{i}+k r_{1}\right)}+\text { c.c. }\right\} \text {. }
$$

$\left({ }^{2}\right)$ Les formules correspondant à une absorption en couche L se trouvent dans l'article de Teo et Lee [38].
On a donc

$$
\mu=\mu_{0}\left\{1+\frac{i f(\pi, k)}{2 k r_{1}^{2}} \mathrm{e}^{2 i\left(\delta_{1}^{\prime}+k r_{1}\right)}+\text { c.c. }\right\}
$$

en ne retenant que les termes du premier ordre, ce qui est justifié par l'intensité faible du signal EXAFS - au mieux quelques pour cent - d'où pour les modulations EXAFS

$$
\begin{aligned}
\chi(k)=\frac{\mu-\mu_{0}}{\mu_{0}}=\frac{-|f(\pi)|}{k r_{1}^{2}} \times \\
\quad \times \sin \left[2 k r_{1}+2 \delta_{1}^{\prime}+\arg f(\pi, k)\right] .
\end{aligned}
$$

Cette formulation très simple implique plusieurs approximations drastiques, notamment le remplacement des fonctions de Hankel par leurs formes asymptotiques et l'assimilation à une onde plane pour le traitement de la rétrodiffusion. Lee et Pendry [13] ont établi une dérivation plus générale qui s'affranchit de ces limitations et doit en principe être utilisée lorsqu'on veut analyser des spectres à des énergies faibles du photoélectron $(E<100 \mathrm{eV})$. Une autre approximation implicite suppose que le potentiel dans l'atome central n'a pas relaxé au cours du processus EXAFS puisqu'on utilise le même déphasage $\delta_{1}^{\prime}$ à l'aller et au retour du photoélectron. Noguera et al. [33] ont montré théoriquement que dans les métaux, un écrantage dynamique du trou 1s par les électrons de conduction pouvait avoir lieu pendant le temps caractéristique du processus EXAFS au moins pour les basses énergies du photoélectron, et un effet systématique de ce type a été observé pour l'aluminium [17]. Enfin, on a fait une théorie de diffusion simple qui repose sur la petitesse du libre parcours moyen électronique dans la matière (Fig. 4) (quelques $\AA ̊$ à $100 \mathrm{eV}$ ) : ici aussi cette approximation n'est pas valable pour de basses énergies du photoélectron $(E<50$ à $100 \mathrm{eV})$. Pour toutes ces raisons, la formule (2) établie ici, et qui est - avec les modifications discutées ci-après - généralement utilisée par les expérimentateurs en EXAFS, ne devrait pas être utilisée sans précaution dans les 100 premiers eV des spectres EXAFS.

Cette formule (2) doit incorporer deux termes d'amortissement avec l'énergie du photoélectron pour être utilisable :

- Un terme décrivant l'amortissement par les effets inélastiques responsables du libre parcours moyen des électrons $\lambda(k): \exp \left[-\frac{2 r}{\lambda(k)}\right]$.

On prend généralement $\lambda(k)=k / \gamma$ avec $\gamma \simeq 4 \mathrm{eV}$ pour des énergies de l'électron supérieures à $50 \mathrm{eV}$. Cette approximation a été discutée théoriquement par Pendry [34] dans un modèle d'interaction du photoélectron avec les électrons de conduction et est en bon accord avec les valeurs expérimentales des libres parcours moyens d'électrons tabulées par Lindau et Spicer [35]. 
- Un terme du type Debye-Waller traduisant l'effet des fluctuations de la distance $R_{1}$. Si $\sigma_{1}$ est l'écart type de la distribution des distances $R_{1}$, il s'écrit $\exp \left(-2 \sigma_{1}^{2} k^{2}\right)$ (et non $\exp \left(-\frac{\sigma_{1}^{2} k^{2}}{2}\right)$ comme dans la diffusion des RX, car en EXAFS le moment transféré est $2 k$ ).

$\sigma_{1}$ mesure la fluctuation des distances entre atomes

$$
\chi(k)=-\frac{1}{k} \sum_{\mathrm{j}} \frac{N_{\mathrm{j}}}{R_{\mathrm{j}}^{2}} \exp \left(-2 \sigma_{\mathrm{j}}^{2} k^{2}\right) \exp -\frac{2 R_{\mathrm{j}}}{\lambda(k)}
$$

L'inspection de la formule (3) montre ce qu'on peut obtenir à partir de l'analyse du spectre EXAFS :

- distance entre atome central et diffuseurs : précision $\sim 0,01 \AA$ dans les bons cas (signal correct jusqu'à $600-700 \mathrm{eV}$ du seuil),

- nombre de diffuseurs $N$ dans la $\ddot{\text { re }}$ couche : précision de 0,2 à 0,5 atome,

- mesure de la fluctuation $\sigma$ de la distance $R$ : précision sur $\sigma^{2}$ de l'ordre de $10^{-2} \AA^{2}$ dans des mesures comparatives,

- identification de l'atome rétrodiffuseur à partir de la phase si on connaît les distances, ou de la variation de l'amplitude du signal avec l'énergie du photoélectron. En effet ces paramètres varient beaucoup avec le numéro atomique. On estime qu'on peut discriminer deux atomes diffuseurs de numéros atomiques différents de 10.

Ces différents points vont être discutés dans le cours de cet article, et notamment au paragraphe suivant où nous montrerons sur un exemple précis les limites de précision de l'EXAFS. Disons tout de suite que la précision dépend de la qualité du signal expérimental, et principalement de son extension en énergie, dans la mesure où la théorie simple de l'EXAFS qui est généralement utilisée est d'autant meilleure qu'on l'applique à des photoélectrons de haute énergie.

L'utilisation de la formule (3) pour la détermination des distances $R_{\mathrm{j}}$ implique la connaissance des termes de déphasages atomiques $2 \delta_{1}^{\prime}(k)+\arg f(\pi, k)=\varphi(k)$. Deux possibilités s'ouvrent alors à l'expérimentateur soucieux d'analyser ses résultats sans calculer luimême ces déphasages. La première est d'utiliser des déphasages théoriques : Lee et al. ont publié des listes de déphasages, ainsi que de modules $|f(\pi, k)|$ pour pratiquement tous les éléments sous forme de courbes en fonction de $k[37,38]$ ou de formules paramétrisées [39, 40]. Ces calculs sont faits pour des atomes libres et doivent être utilisés avec prudence pour des énergies de photoélectron inférieures à $100 \mathrm{eV}$. C. Noguera et D. Spanjaard à Orsay ont également récemment établi des programmes permettant le calcul de ces termes en s'affran- voisins et non, comme dans la diffraction des $\mathrm{X}$ ou des neutrons, l'amplitude de vibration.

Ce terme amortit beaucoup le spectre en fonction de l'énergie du photoélectron, et peut limiter dans certains cas l'emploi de l'EXAFS à des systèmes très désordonnés [36]. Nous y reviendrons plus loin. Finalement, en sommant sur tous les atomes rétrodiffuseurs on obtient la formule maintenant classique et généralement utilisée :

$\left|f_{\mathrm{j}}(\pi, k)\right| \sin \left[2 k R_{\mathrm{j}}+2 \delta_{1}^{\prime}(k)+\arg f_{\mathrm{j}}(\pi, k)\right]$.

chissant de certaines restrictions présentes dans les calculs de Lee (non-écrantage du trou créé dans l'atome central, traitement complet de la rétrodiffusion par des ondes sphériques, utilisation de potentiels calculés pour des solides dans une théorie de muffintin). La seconde consiste à mesurer la phase $\varphi(k)$ dans un corps de structure connue et à transférer ce déphasage expérimental dans l'interprétation de l'EXAFS pour des corps où les distances $R_{\mathrm{j}}$ ne sont pas connues.

Ces deux méthodes impliquent la transférabilité des déphasages pour des états électroniques ou des environnements chimiques différents. Citrin et al. [41] ont testé la validité de la transférabilité des déphasages dans des composés covalents et montré qu'elle était compatible avec une précision de $10^{-2} \AA$ dans la détermination des distances. Elle est plus douteuse lorsqu'on transfère les déphasages à des atomes de degrés d'ionisation différents. Lee et Teo [38] ont calculé dans quelques cas l'influence de l'ionisation et montré qu'elle était de peu d'importance dès que l'énergie du photoélectron dépasse $150-200 \mathrm{eV}$, et pouvait être compensée par une variation du zéro d'énergie des photoélectrons, sur laquelle nous allons revenir. Dans le cas de cristaux ioniques cependant, l'existence de potentiel coulombien en $1 / r$ rend théoriquement caduque l'utilisation de déphasages calculés pour des atomes neutres isolés [42]; pratiquement l'utilisation des déphasages calculés par Lee [37] à l'interprétation de l'EXAFS dans $\mathrm{Al}_{2} \mathrm{O}_{3}$ [17] donne une distance de $1,905 \AA$ pour Al-O, alors que la valeur moyenne est $1,915 \AA$; la précision obtenue est donc encore de $1 / 100 \AA$, alors que le signal expérimental obtenu pour des éléments légers comme $\mathrm{Al}$ et $\mathrm{O}$ est limité à $250 \mathrm{eV}$ au-delà du seuil $\mathrm{K}$, et qu'on est donc dans un cas théoriquement douteux. L'hypothèse de transférabilité des déphasages paraît donc maintenant assez bien fondée du moins lorsqu'on l'associe à un ajustement du zéro $E_{0}$ de l'énergie des photoélectrons. En effet ce zéro d'énergie $E_{0}$ n'est pas expérimentalement bien défini. Il ne coïncide pas a priori avec le seuil d'absorption $\mathrm{X}$ qui donne en fait le niveau de Fermi pour un métal, ou les premiers états vides de symétrie $p$ pour un isolant ou un semi- 
conducteur. Il devrait en fait être situé au fond de la bande de conduction dans un modèle d'électrons presque libres : cela a d'ailleurs été trouvé lors de l'analyse de l'EXAFS de l'aluminium (seuil K) [17] où l'ajustement de $E_{0}$ donne $-12 \mathrm{eV}$ par rapport au niveau de Fermi, donc à $\sim 1 \mathrm{eV}$ du fond de la bande de conduction. Dans la plupart des cas, la position relative de $E_{0}$ et du seuil d'absorption X n'est pas connue, et $E_{0}$ doit être considéré comme un paramètre ajustable. Le moment du photoélectron est évidemment donné, en unités atomiques par :

$$
k=\left\{\left(\hbar \omega-E_{0}\right) / 13,605\right\}^{1 / 2}
$$

où $\hbar \omega$ est l'énergie du photon, et $h \omega$ et $E_{0}$ sont donnés en $\mathrm{eV}$. Une variation de $E_{0}$ revient à dilater les moments

$$
\frac{\Delta k}{k} \sim \frac{1}{2} \frac{\Delta E_{0}}{\hbar \omega-E_{0}} \quad \Delta k=\frac{1}{27,2} \frac{\Delta E_{0}}{\sqrt{E}}
$$

et ce d'autant plus que l'énergie $E$ du photoélectron est plus faible. Elle peut ainsi partiellement compenser d'éventuelles inexactitudes des déphasages à basse énergie. On a donc intérêt lorsqu'on utilise des valeurs calculées pour les déphasages à optimiser le choix de $E_{0}$ de façon à compenser ainsi toutes les imprécisions, ou les inexactitudes que comportent les calculs de déphasage.

1.4 Méthode D'ANAL YSE D'Un SPECTRE EXAFS. Le premier stade consiste à extraire les modulations EXAFS $\chi(k)$ de la courbe d'absorption $\mu(E)$ en fonction de l'énergie : l'absorption gazeuse $\mu_{0}(E)$, qu'il faut soustraire pour obtenir les modulations EXAFS (cf. formule (2)) n'est en général pas connue. On l'approche en lissant le spectre jusqu'à ce que les ondulations EXAFS aient disparu ou par un polynôme de degré ajustable. On choisit une origine d'énergie $E_{0}$ pour les photoélectrons qui soit arbitraire, mais raisonnable, c'est-à-dire près du seuil pour autant qu'on puisse définir sans ambiguité un seuil.

On obtient ainsi des signaux $\chi(E)$, ou $\chi(k)$, semblables à ceux représentés sur la figure 7 : ces signaux ont été obtenus pour une concentration atomique de $1 \%$ de cuivre dans une matrice d'aluminium [43], ce qui illustre la sensibilité de l'EXAFS. Le spectre $7 a$ est relatif à une solution solide de cuivre dans $\mathrm{Al}$, et la figure $7 b$ à une phase $\theta^{\prime}\left(\mathrm{Al}_{2} \mathrm{Cu}\right)$ dont la structure a été déterminée par diffraction des $\mathrm{RX}$ et qui va servir de référence permettant de tester la validité de l'analyse, et notamment de déterminer la valeur de $E_{0}$. Il est important que la phase de référence ait des structures, chimique et cristallographique, les plus proches possibles de celles des phases qu'on souhaite déterminer, pour que l'on puisse transférer $E_{0}$ et les déphasages d'une situation à une autre sans problème.

L'inspection de la formule (3) montre que $k \chi(k)$

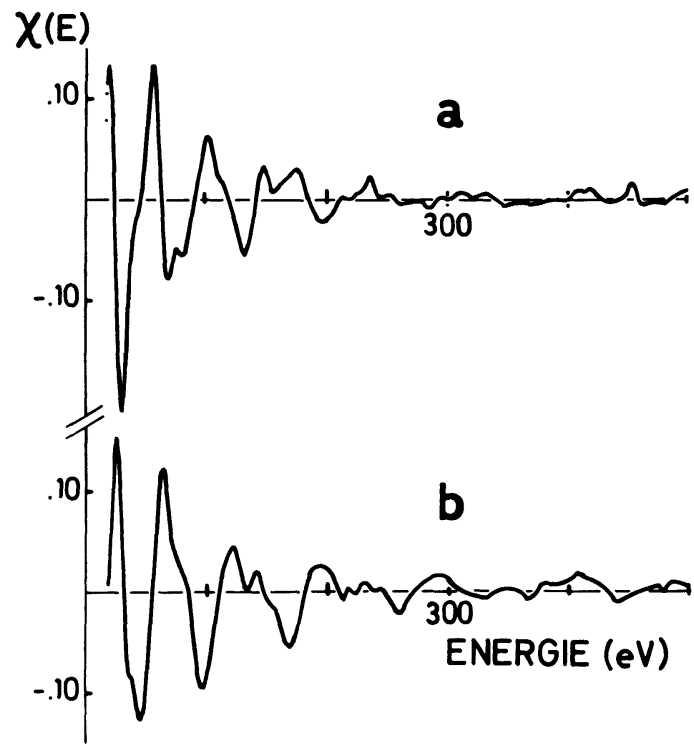

Fig. 7. - Spectres EXAFS au seuil K du cuivre : $a$ ) d'une solution solide de $2 \%$ atomique de $\mathrm{Cu}$ dans $\mathrm{Al}, b$ ) de la phase $\theta^{\prime} \mathrm{Al}_{2} \mathrm{Cu}$ (même composition globale qu'en $a$ )).

[EXAFS signals $\chi(k)$ above the copper $\mathrm{K}$ edge for : $a$ ) a solid solution of $2 \%$ atomic concentration of $\mathrm{Cu}$ in a $\mathrm{Al}$ matrix, $b$ ) precipitates of the $\theta^{\prime}$ phase $\left(\mathrm{Al}_{2} \mathrm{Cu}\right.$ ) in a $\mathrm{Al}$ matrix (same $2 \%$ atomic concentration of $\mathrm{Cu})$.]

est proportionnel à $\sin (2 k R+\varphi(k))$. Il est donc naturel d'effectuer une transformation de Fourier qui va donner, dans l'espace réel, les pics d'une fonction de distribution de paires (cf. Fig. 8). Ces pics sont déplacés par rapport à la vraie valeur $R$ à cause de la phase $\varphi(k)$. Dans les premières analyses d'EXAFS, Stern et al. $[9,10,12]$ ont supposé que la phase atomique dépendait linéairement de $k, \varphi(k)=\alpha k+\beta$. Les pics des transformées de Fourier donnent des distances

$$
R_{\text {apparent }}=R+\frac{\alpha}{2} \text {. }
$$

Ils ont montré que, généralement $\alpha / 2$ varie de $-0,3$ à $-0,4 \AA$. Cette constatation est en fait indépendante de la linéarité de $\varphi(k)$ en $k$, qui n'est qu'approximative. Dans ce cas présent, on observe 2 pics pour la phase $\theta^{\prime}$ qui correspondent à la couche des premiers voisins $\mathrm{Cu}-\mathrm{Al}$, et à celle des deuxièmes voisins $\mathrm{Cu}-\mathrm{Cu}$. On obtient pour la première couche, une distance apparente de $2,13 \AA$, alors que par diffraction des X on obtient $2,487 \AA$, soit une translation de $0,36 \AA$, qui est bien de l'ordre de grandeur attendu. Si l'on admet la validité de la transférabilité des déphasages, on peut se contenter de comparer la valeur à laquelle pique la transformée de Fourier du spectre correspondant à la solution solide de cuivre dans l'aluminium - pour laquelle on ne connaît pas la distance $\mathrm{Cu}-\mathrm{Al}$ dans la première couche - à la distance apparente dans la première couche de la phase $\theta^{\prime}$. On obtient un déplacement de $+0,24 \AA$ visible sur la figure 8 , d'où une mesure directe de la distance à la 


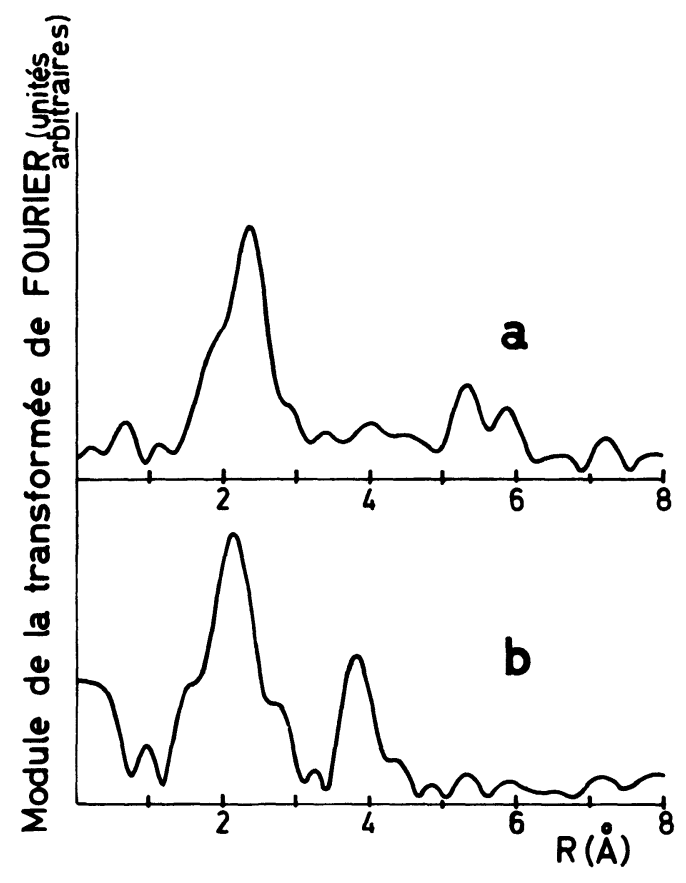

Fig. 8. - Amplitudes des transformées de Fourier des $k \chi(k)$ pour : $a$ ) la solution solide, $b$ ) la phase $\theta^{\prime}$.

[Absolute values of the Fourier transforms of $k \chi(k)$ for the spectra shown in figure $7: a$ ) solid solution, $b$ ) $\theta^{\prime}$ phase.]

première couche de voisins dans la solution solide : $\mathrm{CuAl}=2,487+0,24 \sim 2,73 \AA$ (contre 2,85 dans la matrice d'aluminium). Cette valeur est très proche de la valeur optimale $2,725 \AA$; cet exemple montre qu'une simple comparaison des transformées de Fourier correspondant aux signaux d'EXAFS d'une phase inconnue et d'une phase de référence suffit souvent à mesurer les distances dans la phase inconnue : encore faut-il que ces 2 phases soient très semblables (même composition chimique, distances entre voisins proches, et facteurs Debye-Waller $\sigma$ voisins, $\Delta \sigma<0,01 \AA)$.

On est cependant amené, en général, à faire une analyse plus fine. C'est notamment le cas lorsque deux, ou plusieurs, atomes différents figurent dans la même couche et donnent des contributions déphasées les unes par rapport aux autres. Par ailleurs, si l'on veut mesurer le nombre $N_{\mathrm{j}}$ d'atomes dans une couche, ou apprécier l'amortissement $\sigma^{2}$ du spectre, c'est-àdire si on travaille sur l'amplitude du signal, on ne peut se contenter d'une comparaison de fonctions de distributions de paires. La méthode suivie alors consiste à reproduire le spectre expérimental en utilisant la formule (3) et des déphasages théoriques; dans le cas présent, on a utilisé des déphasages calculés par D. Spanjaard et C. Noguera. Mais auparavant, on utilise une technique de filtrage qui isole le pic dans la transformée de Fourier correspondant à la couche considérée. On le transforme ensuite en sens inverse pour obtenir ainsi un signal d'EXAFS partiel correspondant à une seule couche, ce qui permet de limiter le nombre de paramètres à ajuster

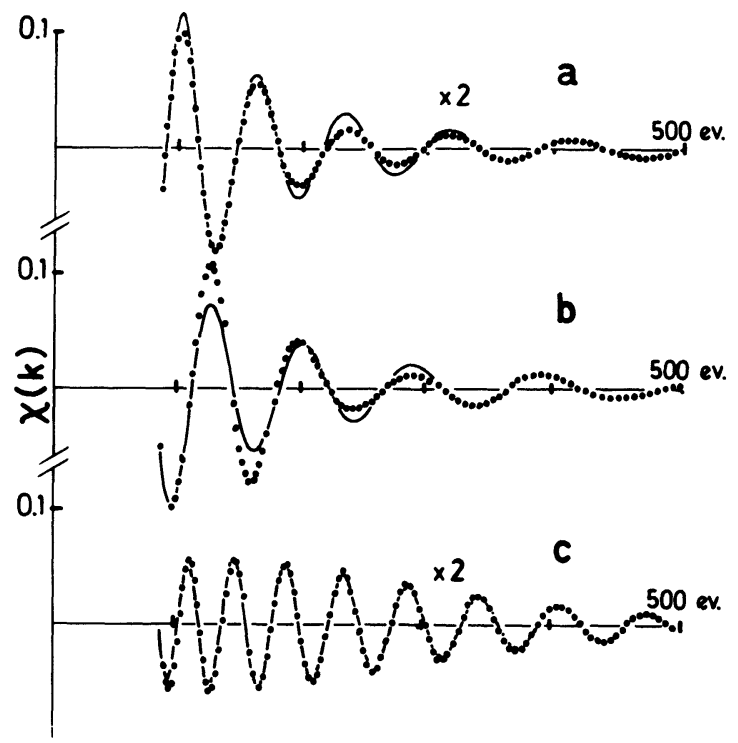

Fig. 9. - Filtrage de Fourier : a) première couche $\mathrm{Cu}-\mathrm{Al}$ dans la solution solide, $b$ ) première couche $\mathrm{Cu}-\mathrm{Al}$ dans la phase $\theta^{\prime}, c$ ) deuxième couche $\mathrm{Cu}-\mathrm{Cu}$ dans la phase $\theta^{\prime}$ (les résultats expérimentaux sont représentés par des points... les courbes continues sont la reconstitution théorique).

[Fourier filtering of the EXAFS spectra shown in figure 7 (dotted curves) and theoretical fits (solid curves) : a) first coordination sphere (Al neighbours) around a copper atom in the solid solution, $b$ ) idem but in the $\theta^{\prime}$ phase, $c$ ) second coordination sphere ( $\mathrm{Cu}$ atoms) in the $\theta^{\prime}$ phase.]

(Fig. 9). Ce nombre de paramètres est d'ailleurs réduit du moints tant qu'on ne travaille que sur la phase du signal : les déphasages étant calculés indépendamment, on a seulement 2 paramètres libres $R_{\mathrm{j}}$ et le zéro d'énergie $E_{0}$. En fait le choix de $E_{0}$ n'est pas indépendant des déphasages atomiques et peut être déterminé de façon systématique. Une première méthode proposée par Lee et Beni [37] consiste à multiplier les données par

$$
\exp \left[-i\left(\arg f(\pi, k)+2 \delta_{1}^{\prime}(k)\right)\right]
$$

où $k$ est défini par le choix de $E_{0}$. On effectue la transformation de Fourier complexe et, pour le bon choix de $E_{0}$, le maximum de la partie réelle de la T.F. doit coïncider avec une annulation de la partie imaginaire, puisque ces 2 signaux sont alors les transformées de Fourier réelle et imaginaire de $\sin (2 k R)$. Une autre méthode suggérée initialement par Rabe et al. [44] consiste à écrire qu'aux nœuds des signaux d'EXAFS partiels tels ceux représentés sur la figure 9 on a la relation :

$$
2 k_{n} R_{n}+\varphi\left(k_{n}\right)=\left(n_{0}+n\right) \pi
$$

où $n$ désigne le numéro du nœud.

Pour chaque choix de $E_{0}$, on obtient ainsi un certain nombre de déterminations $R_{n}$ de $R$ qui en principe ne devraient pas dépendre de $n$ si $E_{0}$ est correctement déterminé. C'est cette méthode que nous avons utilisée (cf. Fig. 10). On mesure l'écart type $\sigma^{2}$ de la 


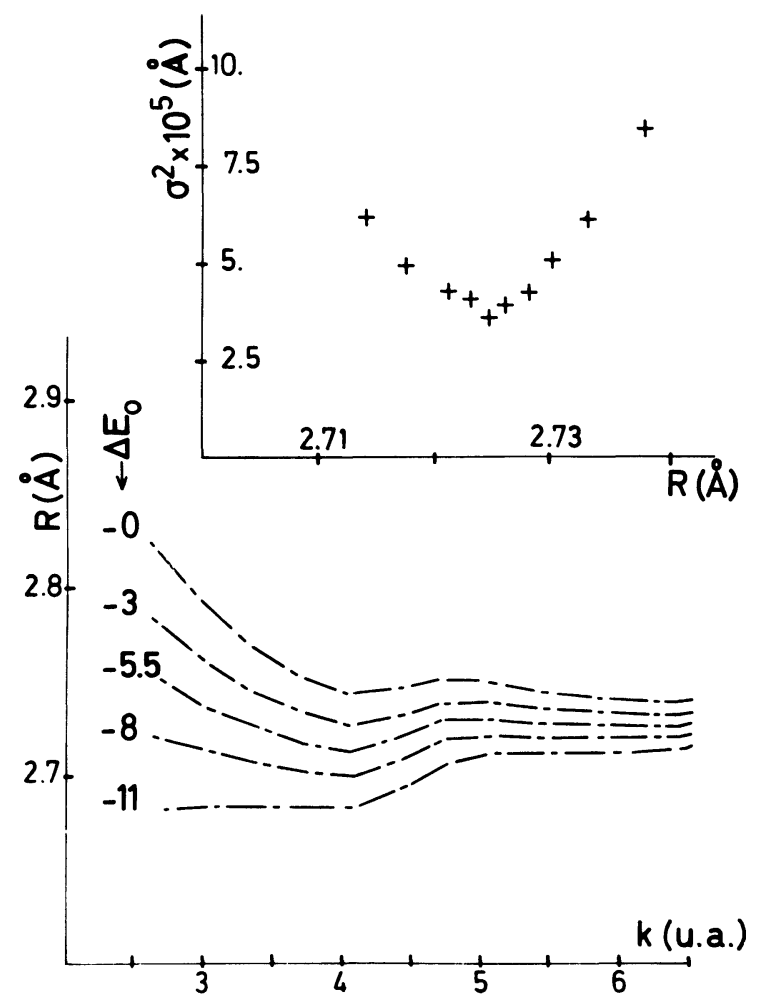

Fig. 10. - Détermination des distances $\mathrm{Cu}-\mathrm{Al}$ à partir des positions des zéros sur les courbes expérimentales filtrées (Fig. 9) pour diverses valeurs $E_{0}$ de déplacement du seuil. Dans la partie supérieure, écart quadratique de la distribution des distances en fonction de la valeur moyenne pour diverses valeurs de $E_{0}$.

[Determination of the first neighbour distance from the zeros of the Fourier filtered signal for the $\mathrm{Cu}: \mathrm{Al}$ solid solution. The threshold energy $E_{0}$ is varied around the $\mathrm{K}$ edge energy, and for each $E_{0}$ value, one gets a set of $R(k)$ distances determined at each node of the signal. The $R(k)$ curves are shown in the lower part. The mean values $\bar{R}$ and the standard deviation $\sigma$ are calculated and the $\sigma$ values are plotted as a function of $\bar{R}$ for different $E_{0}$ values in the upper part : the best $R$ value minimizes $\sigma$.]

distribution des $R_{n}$ autour de la valeur moyenne $\bar{R}$, pour différents choix de $E_{0}$. La variation de $\sigma^{2}$ en fonction de $\bar{R}$ est en fait une courbe très raide qui permet de déterminer la valeur optimale de $\bar{R}$ avec une précision, dans le cas présent de $0,01 \AA$. On a ainsi obtenu $E_{0}=-8 \mathrm{eV}$ par rapport à l'inflexion $\mathrm{du}$ seuil d'absorption $\mathrm{K}$ du cuivre pour la phase $\theta^{\prime}$ comme pour la solution solide. Dans le cas de la phase $\theta^{\prime}$, cette procédure donne $\bar{R}=2,48 \AA$ pour la première couche et $4,06 \AA$ pour la seconde; l'accord est donc à mieux de $0,007 \AA$ avec les résultats de diffraction des rayons $X(2,487$ et $4,06 \AA)$.

La figure 9 permet de comparer les spectres filtrés aux reconstitutions théoriques effectuées en utilisant la formule (3). L'accord est très bon en ce qui concerne les phases, ce qui justifie bien la procédure que nous avons utilisée. Il est un peu moins bon en ce qui concerne les amplitudes, notamment dans la région $200-300 \mathrm{eV}$; ce qui vient probablement de la difficulté qu'il y a à rendre compte dans le détail des amplitudes lorsqu'on utilise dans le terme d'amor- tissement un libre parcours moyen électronique linéaire en $k$ et donc notablement différent de la forme de la courbe dite universelle représentée sur la figure 4. D'autres effets liés à des processus intraatomiques dans l'atome central peuvent aussi affecter de 20 à $30 \%$ ces amplitudes [45].

En conclusion, cet exemple illustre bien la précision que l'on peut attendre de l'analyse d'une expérience d'EXAFS, à savoir typiquement $1 / 100$ d'angströms, et ce même dans un mauvais cas, comme celui que nous avons détaillé ici (faible teneur en $\mathrm{Cu}$, faible rétrodiffusion par les voisins aluminium légers pour $E>200 \mathrm{eV}$ ).

Une telle précision est cependant plus difficile à obtenir pour une couche formée d'atomes de plusieurs types ou à des distances différentes de l'atome absorbant [47]. Ce point sera discuté en 2.1.

2. L'EXAFS dans les milieux désordonnés. Jusqu'en 1976-77, la littérature consacrée à l'EXAFS portait essentiellement sur des études, théoriques ou expérimentales, dont le but était d'établir et de vérifier les bases de l'interprétation; elles étaient donc faites dans des milieux simples, de structure locale totalement ou assez bien connue par d'autres méthodes. L'EXAFS devient maintenant un outil de détermination structurale sur des systèmes plus ou moins complexes. La plus grande partie de ces travaux ont été faits sur des systèmes désordonnés ; ce fait s'explique bien puisque, on l'a vu, l'EXAFS garde ses possibilités dans ces milieux où les autres méthodes sont souvent inutilisables.

Nous décrivons dans cette partie les principaux résultats publiés sur les solides amorphes (2.1) et les liquides (2.2). Une présentation des études de solides cristallisés présentant des types variés de désordre et une discussion des limitations de l'EXAFS dans ces milieux est faite en 2.3.

Signalons qu'une première revue des études d'EXAFS dans les milieux désordonnés a été faite par T. M. Hayes en 1978 [49].

2.1 ETUDES STRUCTURALES D'AMORPHES. - Nous reprenons ici séparément les paramètres structuraux impliqués dans la structure EXAFS pour discuter, sur chacun, les renseignements qu'apporte cette spectroscopie.

Distances interatomiques. - Le plus souvent, dans les amorphes, seule la première couche de voisins donne une structure EXAFS significative, les distributions des distances dans les couches suivantes étant trop larges pour donner une structure d'interférences visible $(\Delta R \gtrsim 0,2)$.

$\mathrm{Ce}$ fait est visible au premier examen, que l'on regarde le spectre $k \chi(k)$ ou sa transformée de Fourier $f(R)$, c'est-à-dire les pics de la distribution radiale : le spectre $k \chi(k)$ est dominé par la seule fréquence correspondant à la distance entre premiers voisins contrairement au mélange de fréquences apparais- 
sant sur les spectres de corps cristallisés ; la transformée de Fourier $f(R)$ présente un seul pic.

Les spectres $\chi(k)$ et $f(R)$ réalisés, au seuil du fer, dans des verres silicatés dopés au fer et dans des composés cristallisés du fer, présentés figure 11 sont très significatifs à ce sujet [31].

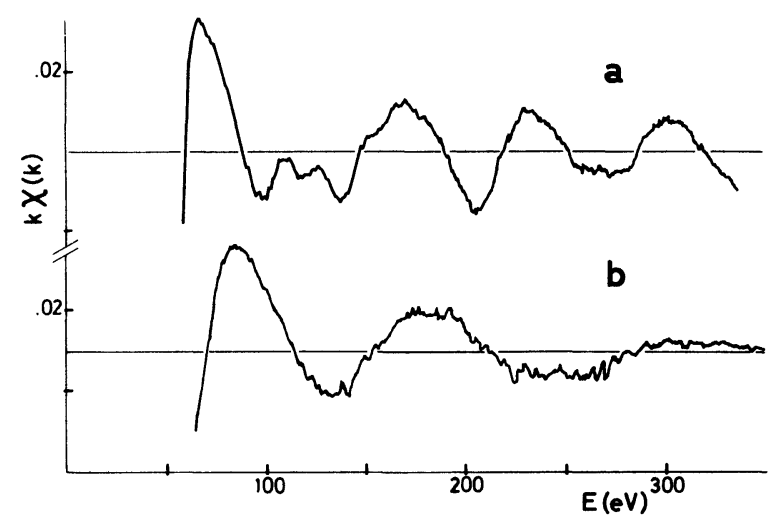

Fig. 11. - Structures d'absorption au seuil $\mathrm{K}$ du fer : $a$ ) oxyde ferrique $\mathrm{Fe}_{2} \mathrm{O}_{3}, b$ ) un verre synthétique de composition $\mathrm{Na}_{2} \mathrm{O}$, $2 \mathrm{SiO}_{2}$ dopé au fer et élaboré en milieu oxydant.

[EXAFS signal on the iron $\mathrm{K}$ edge for : a) $\mathrm{Fe}_{2} \mathrm{O}_{3}, b$ ) a synthetic glass, $\mathrm{Na}_{2} \mathrm{O} 2 \mathrm{SiO}_{2}$, doped with iron in an oxidizing environment.]

A propos du germanium amorphe, qui a été étudié par plusieurs équipes, les résultats sont contradictoires mais traduisent sans doute des différences d'amorphicité des échantillons : dans les spectres réalisés par S. Hunter [49], seuls les premiers voisins contribuent à l'EXAFS alors que D. E. Sayers et al., dans des mesures antérieures [51], observaient une contribution faible mais mesurable des seconds voisins. L'élargissement du pic révélerait une distribution des distances radiales de $\sim 0,15 \AA$ sur cette couche.

Les informations données par l'EXAFS sur la distribution radiale de couches successives doivent évidemment être comparées à celles qu'apporte la diffraction sur l'éventuelle cristallinité de ces couches. Sayers et al. [52-53] ont beaucoup étudié cette question sur $\mathrm{GeO}_{2}$ et $\mathrm{GeSe}$, systèmes très étudiés aussi par diffraction des rayons $\mathrm{X}$ et des neutrons; dans $\mathrm{GeO}_{2}$ l'accord entre les méthodes est bon pour les premières $(\mathrm{Ge}-\mathrm{O})$ et secondes $(\mathrm{Ge}-\mathrm{Ge})$ couches. Les distances $\mathrm{Ge}-\mathrm{O}$ de la première couche et Ge-Ge sont inférieures à celles de $\mathrm{GeO}_{2}$ en phase hexagonale de $\sim 0,05$ et $0,07 \AA$.

Dans GeSe, l'EXAFS a apporté des informations riches parce que les spectres ont pu être faits aux seuils $\mathrm{K}$ des 2 composants, Ge et Se. Cette situation est toujours très favorable évidemment : dans le travail de S. Hunter [54, 55] sur les amorphes $\mathrm{Cu}_{x}\left(\mathrm{As}_{2} \mathrm{~S}_{3}\right)_{1-x}$, beaucoup de renseignements ont pu être tirés grâce au fait que les seuils des 3 composants étaient accessibles à Stanford; on peut citer aussi les possibilités qui sont exploitées actuellement à LURE dans des oxydes et oxysulfures mixtes de gallium et de terre rare où sont mesurés les spectres au seuil $\mathrm{K}$ de Ga et aux seuils $\mathrm{L}$ de la terre rare.

Distribution radiale. - Les désordres statique ou dynamique dans chaque couche élargissent les pics dans l'espace réel; le terme $\mathrm{e}^{-2 \sigma_{j}^{2} k^{2}}$ de la formule (3) rend compte de cette distribution radiale des distances interatomiques.

Dans les amorphes, cette distribution, pour la première couche n'est généralement pas plus étalée que dans les cristaux. Dans $\mathrm{As}_{2} \mathrm{Te}_{2}$ vitreux, par exemple Pettifer et al. [50] trouvent des pics plus étroits que dans le cristal.

La distribution est au contraire large pour les couches atomiques suivantes au point, on l'a vu, de supprimer la contribution de ces couches à la structure EXAFS.

Nature et nombre des premiers voisins. - Trois travaux illustrent bien les possibilités de l'EXAFS pour ces problèmes : la détermination de la coordination du cuivre, de l'arsenic et du sélénium dans les alliages $\mathrm{Cu}_{x}\left(\mathrm{As}_{2} \mathrm{Se}_{3}\right)_{1-x}$ par S. Hunter [54, 55], celle du germanium dans les amorphes $\mathrm{Pd}_{\sim 0,80} \mathrm{Ge}_{\sim 0,02}$ par T. M. Hayes et al. [56] et celle de l'arsenic dopant des alliages Si-H amorphes par J. C. Knights et al. [57]. L'étude de $S$. Hunter démontre particulièrement bien l'avantage éventuel de l'EXAFS par rapport aux déterminations de positions de distribution radiale par diffraction : dans un amorphe à 3 composants de poids atomiques voisins, les nombres de coordination relatifs à chacun sont déterminés séparément sans ambiguité alors que la diffraction donnerait une information moyenne. Avec une précision d'environ 0,5 sur le nombre de coordination, il est montré que le cuivre garde la même coordination dans les alliages amorphes que dans les composés cristallisés, alors que le nombre de coordination de l'arsenic et surtout celui du sélénium augmentent avec la concentration en cuivre ( 2 à 4 pour Se en passant du composé à $5 \% \mathrm{Cu}$ au composé à $25 \% \mathrm{Cu}$ ).

Dans les amorphes $\mathrm{Pd}_{0,80} \mathrm{Ge}_{0,20}$, Hayes et al. [56] ont obtemu sur l'EXAFS mesuré au seuil de $\mathrm{Ge}$, la contribution pratiquement unique des premiers voisins Pd : l'amplitude de l'EXAFS donne un nombre de voisins égal à $8,6 \pm 0,5$; la largeur du pic dans l'espace réel montre que la distribution radiale sur cette couche est étroite $(<0,1 \AA)$. Les mesures prouvent aussi l'inexistence des voisins Ge-Ge. Le nombre et la nature des voisins du germanium ainsi déterminés sont en accord avec l'hypothèse d'un ordre chimique et les résultats de diffusion des rayons $\mathrm{X}$ de J. F. Sadoc et J. Dixmier [58]. L'étroitesse de la distribution des distances Ge-Pd s'accorde mal par contre avec un arrangement désordonné compact des atomes métalliques, les atomes de germanium se plaçant dans les vides (de coordination 8 et 9) de cet empilement ; ce modèle conduit à un élargissement bien supérieur à celui qui est observé pour les distances Pd-Ge. 
Le cas des alliages Si-H dopés à l'arsenic [57] est caractéristique quant à lui des possibilités de l'EXAFS dans les systèmes dilués; avec une concentration atomique en As de $1 \%$ à $10 \%$, Knights et al. ont pu faire une étude précise de la première couche de voisins : les plus proches voisins de l'arsenic sont des atomes de silicium ; la précision donnée est très grande sur la distance moyenne et le nombre de ces voisins déterminés par comparaison avec As- $\mathrm{H}$ amorphe $(2,80 \pm 0,04$ voisins à $2,38 \pm 0,01 \AA$ pour l'alliage dopé à $1,7 \%$ par exemple). Une telle précision sur le nombre d'atomes voisins implique que tous les autres paramètres, notamment le libre parcours moyen électronique et la largeur de distribution radiale, soient identiques dans le système étudié et la référence, ce qui est peut-être à prouver. La coordination de l'arsenic décroît légèrement avec le taux de dopage. La seconde couche ne contribue pas dans ce cas encore à la structure EXAFS, et les auteurs concluent à une distribution $>0,2 \AA$ sur cette couche.

Il faut rappeler que les déterminations du nombre de coordination reposent sur la mesure de l'amplitude de l'EXAFS dont la difficulté est plus grande que celle de la périodicité des structures : la présence, dans le faisceau monochromatisé, des harmoniques de la réflexion utilisée et les problèmes d'homogénéité d'échantillon sont les deux points délicats. A. E. Stern et al. [59] ont été amenés, pour ces raisons, à revenir sur des mesures antérieures sur le brome et ont discuté à ce propos les causes d'incertitude sur les amplitudes.

Au sujet de la pollution du faisceau par des harmoniques, signalons qu'au LURE-DCI, l'utilisation de monochromateurs différents, travaillant sur des réflexions (220), ou (111) permet déjà d'éliminer les harmoniques les plus gênants.

Citons en dernier lieu un exemple à propos duquel il existe une divergence entre les conclusions de l'EXAFS et les mesures de diffusion X. Il s'agit de l'étude de films minces de $\mathrm{Nb}_{3} \mathrm{Ge}$ : Brown et al. [74] trouvent dans la phase amorphe une distance $\mathrm{Ge}-$ $\mathrm{Nb}$ égale à 2,66 $\AA$, la coordination des germanium étant passée de 12 (dans la phase A15) à $8 \pm 2$. Ce résultat s'explique par une augmentation de la covalence dans la phase amorphe. Gargill, par des mesures comparatives de diffusion $\mathrm{X}$ entre $\mathrm{Nb}_{3} \mathrm{Ge}$ et $\mathrm{Nb}_{3} \mathrm{Si}$, conclut à une distance $\mathrm{Nb}-\mathrm{Ge}=2,75 \AA$, l'écart est supérieur aux précisions prévues pour chacune des techniques.

De ces exemples, on peut conclure, tout en devant rester prudent étant donné le petit volume de la littérature encore disponible, que l'EXAFS est, dans les amorphes, un bon outil pour l'étude de la première couche de voisins de chacun des éléments aux seuils desquels la mesure peut être faite, les distances interatomiques correspondantes, la largeur de la distribution radiale, son contenu en nature et en nombre peuvent être atteints en général avec une meilleure finesse que par les méthodes de diffraction.

L'EXAFS par contre ne contient le plus souvent aucune information sur les couches suivantes à cause de la trop grande largeur de la distribution radiale sur ces couches.

2.2 Structure locale Des Liquides. - Il existe encore peu de travaux sur les métaux ou sels fondus, probablement parce qu'ils nécessitent des dispositifs expérimentaux (fours avec fenêtres transparentes aux $\mathrm{X}$ ) qui ne sont pas réalisés dans les centres de rayonnement synchrotron. Deux études sur $\mathrm{As}_{2} \mathrm{Se}_{3}$ [60] et $\mathrm{ZnCl}_{2}$ [61] ont mesuré la dépendance du spectre EXAFS avec la température dans les phases amorphes et liquides à travers les températures de recristallisation et fusion. Aucune variation de distance n'a été observée. Par contre à la fusion, on observe une faible diminution de l'écart type $\sigma$ des fluctuations de distance entre atomes proches voisins, qui implique une amélioration de l'ordre local dans la première couche. Dans un domaine voisin, Boyce et al. [62] ont montré les possibilités d'application à l'étude de superconducteurs ioniques (AgI) ; l'interprétation des spectres dans les phases conductrices peut cependant être délicate du fait de l'amortissement très rapide des spectres avec l'énergie lié à la grande mobilité des ions conducteurs (cf. discussion $\S 2.3$ ).

Par contre, l'étude de la structure locale des solutions de sels du type $\mathrm{Ni}^{-\mathrm{Cl}_{2}}$ dans l'eau [46, 63, 64, $65,69]$ ou d'autres solvants [66] a fait l'objet de nombreux travaux qui ont permis de mesurer directement les distances et les nombres d'hydratation dans des solutions diluées à $10^{-2}$ molaires [63] ou concentrées $[46,64,65,66,69]$. De telles mesures sont impossibles par diffusion des $\mathrm{X}$ du fait du fond continu intense dû au solvant. Dans le cas des solutions aqueuses concentrées de bromures de métaux divalents $\mathrm{CuBr}_{2}$ [46], $\mathrm{ZnBr}_{2}$ [65], des changements structuraux étonnants ont été observés en fonction de la concentration, et ce à partir de concentrations de l'ordre du cinquième de la saturation.

La figure $12 a$ montre les signaux EXAFS obtenus pour une poudre de $\mathrm{ZnBr}_{2}$ (1), une solution très concentrée (2) et une solution diluée (3) : les spectres (1) et (2) sont très semblables pour des énergies supérieures à $100 \mathrm{eV}$; ce qui montre très directement l'existence de liaisons $\mathrm{Zn}-\mathrm{Br}$ dans la solution à des distances très proches de celles du cristal. Dans la figure $12 b$, on a tracé les modules des transformées de Fourier pour une solution très diluée, où on observe le pic d'hydratation correspondant à une liaison $\mathrm{Zn}-\mathrm{O}$, pour une solution concentrée où on observe une réduction de ce pic d'hydratation et l'apparition d'un deuxième pic, et enfin pour la poudre cristalline. Le deuxième pic dans la transformée de Fourier du spectre de la solution concentrée coïncide avec celui associé aux liaisons $\mathrm{Zn}-\mathrm{Br}$ du cristal, et les intensités sont tout à fait comparables, ce qui prouve sans aucun besoin d'analyse fine, l'existence de domaines quasi solides dans ces solutions concentrées. Une analyse complète montre qu'au moins $80 \%$ des atomes de soluté sont pris dans cet ordre local, qu'il n'y a proba- 


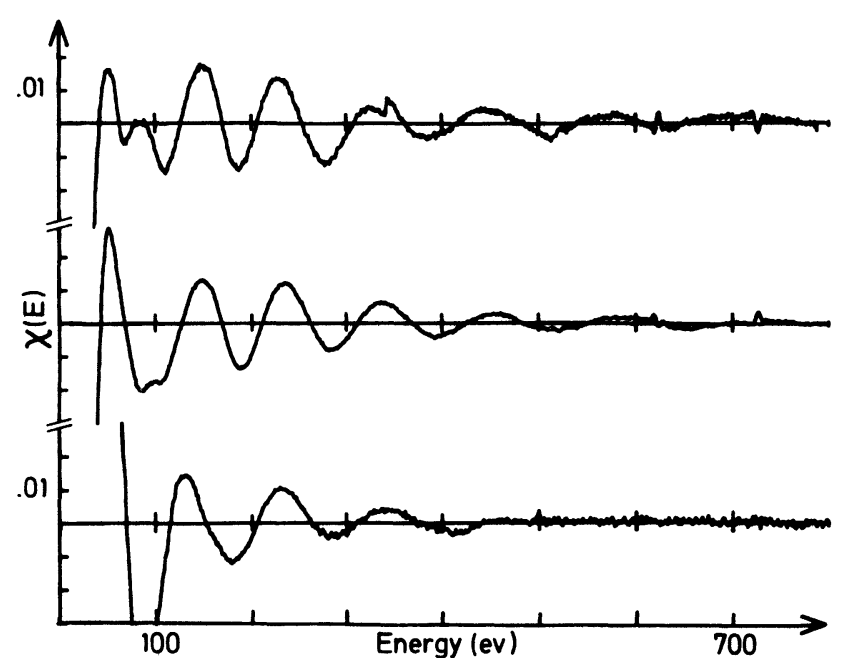

a)

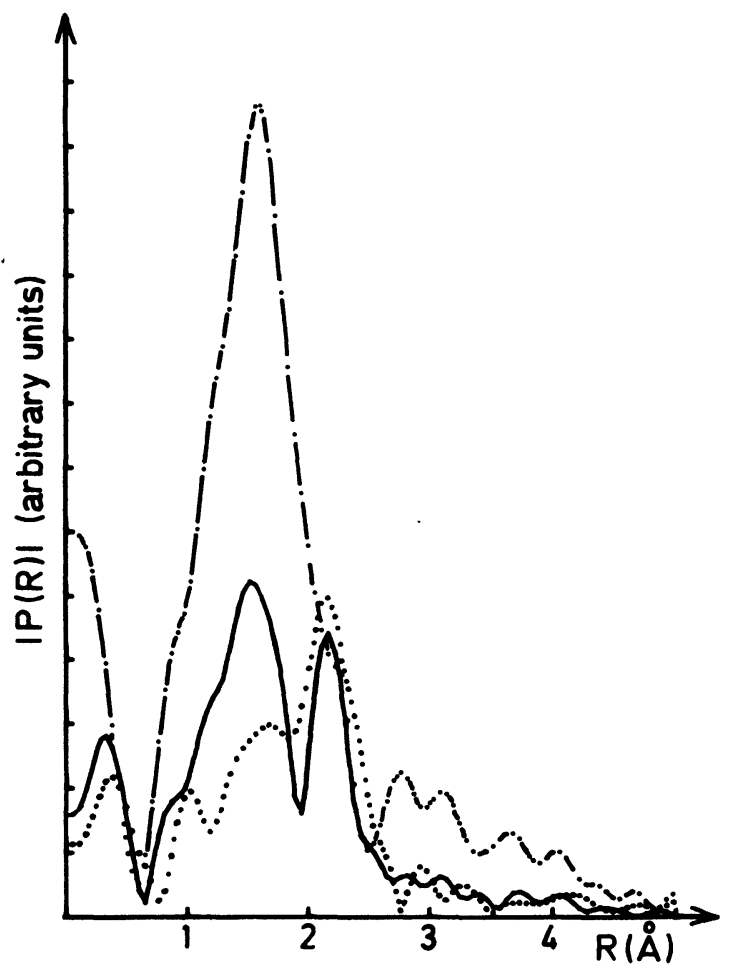

b)

Fig. 12. - a) Spectre EXAFS de $\mathrm{ZnBr}_{2}$ au seuil du zinc : 1) dans le cristal anhydre, 2) dans la solution aqueuse saline $(8 \mathrm{M}), 3)$ dans la solution aqueuse diluée $(0,09 \mathrm{M}) ; b$ ) Amplitudes de transformées de Fourier correspondantes : - solide, .... solution $8 \mathrm{M}$, .- - - solution $0,09 \mathrm{M}$.

[a) EXAFS signals on the zinc $\mathrm{K}$ edge for : 1) an anhydrous $\mathrm{ZnBr}_{2}$ crystal, 2) a heavily concentrated aqueous solution of $\mathrm{ZnBr}_{2}$ $(8 \mathrm{M}), 3)$ a dilute solution $(0,09 \mathrm{M}) ; b$ ) Absolute values of the Fourier transforms of the signals shown in figure $12 a:$ - crystal, .... concentrated $8 \mathrm{M}$ solution, ._. .- dilute $(0.09 \mathrm{M})$ solution.]

blement plus d'eau dans ces domaines ordonnés, et que la structure locale y est presque celle du solide anhydre. En effet la coordination y est la même, conduisant à des unités tétrahédrales de $\mathrm{ZnBr}_{4}$; les distances $\mathrm{Zn}-\mathrm{Br}$ sont de $2,37 \pm 0,02 \AA$ dans les solutions et de $2,403 \AA$ dans le solide anhydre. Les facteurs Debye-Waller qui mesurent le désordre local radial sont eux aussi les mêmes pour les solutions très concentrées et le cristal $\left(\sigma \sim 8 \times 10^{-2} \AA\right)$. On peut donc imaginer ces liquides comme formés de petits domaines solides, vraisemblablement anhydres en volume et hydratés sur leur surface, qui ont une durée de vie limitée, et qui sont séparés les uns des autres par un peu d'eau libre.

Tandis que $80 \%$ des atomes de $\mathrm{Zn}$ ou $\mathrm{Br}$ sont pris dans ces domaines quasi solides dans le cas des solutions concentrées de $\mathrm{ZnBr}_{2}$, environ $50 \%$ restent libres, et seulement hydratés, dans le cas de $\mathrm{CuBr}_{2}$. Puis, pour $\mathrm{NiBr}_{2}$ et $\mathrm{SrBr}_{2}$ [65] on n'observe pas d'ordre dû à des liaisons métal-halogène. La même évolution avec la nature des cations s'observe aussi pour les solutions de chlorure. Il y a donc une grande richesse des structures de ces solutions, qui n'est pas à l'heure actuelle comprise par des considérations simples de physico-chimie. Peut-être vaut-il la peine ici de souligner que ce sont les expériences d'EXAFS qui donnent les premiers modèles structuraux, confirmant cependant les observations faites en diffusion Raman de la lumière qui avaient montré préalablement l'existence de vibrations associées à des liaisons métal-chlore; et ce, pour souligner que l'EXAFS est une technique directe et riche pour étudier des systèmes complexes dans lesquels on cherche à mettre en évidence des effets physiques, ici l'existence d'un ordre à moyenne distance quasi solide dans des liquides, plutôt qu'à mesurer très précisément des paramètres.

2.3 AUtRes EXEMPLES D'EMPLOI. LIMITATIONS POUR LES MILIEUX TRÈS DÉSORDONNÉS. - L'EXAFS est maintenant utilisée dans des domaines divers qui tous relèvent de la physique ou de la chimie des milieux mal cristallisés (on ne parlera pas ici des applications à la biologie : pour des références, voir $[15,72]$ ). Un domaine très actif est celui de la catalyse, références en $[15,73]$ : citons seulement des travaux sur des petites particules de platine dans des zéolites (12 ̊̊ de diamètre) effectués à LURE par Moraweck et al. [67] qui montrent une diminution de la distance entre proches voisins de $0,12 \AA$ par rapport au métal massif, et des variations de distance suivant la couverture par une atmosphère inerte ou par de l'hydrogène. Apai et al. [68] à Stanford ont également observé très récemment une telle relaxation dans des petits agglomérats de $\mathrm{Cu}$ ou Ni. L'intérêt de l'EXAFS est ici sa sélectivité qui permet d'effacer les contributions dues au support, et son caractère de sonde très locale qui permet d'étudier de tout petits agglomérats. Pour les mêmes raisons, l'EXAFS devrait devenir une technique très utile pour l'étude des coeurs de défauts pour laquelle il n'existe pas d'autre technique directe de détermination des relaxations locales. A notre connaissance, les seuls travaux effectués à ce 
jour dans ce domaine concernent des impuretés dans des métaux ( $\mathrm{Cu}$ et $\mathrm{Zn}$ dans $\mathrm{Al}$ ) et des défauts plans étendus, les zones Guinier-Preston [43]. Dans le cas des solutions solides de $\mathrm{Cu}$ dans $\mathrm{Al}$ (cf. § 1.4) on a pu montrer que la distance entre premiers voisins $\mathrm{Cu}$-Al est de 2,725 $\pm 0,015 \AA$, alors qu'elle est de $2,865 \AA$ dans la matrice d'aluminium. Cette première mesure de l'effet de cour autour d'un défaut atomique a conduit à réexaminer l'interprétation habituellement donnée des variations de paramètres en fonction de la concentration en défauts.

Un dernier champ d'application voisin est celui de la détermination de structures chimiques dans des composés non cristallisables, comme l'oxalate de cuivre [70] étudié à LURE par Michalowicz et al. qui ont pu discriminer entre les deux structures proposées pour expliquer l'antiferromagnétisme de ce composé.

Un dernier exemple permet de discuter les limites d'emploi de l'EXAFS dans le cas de systèmes très désordonnés. Il s'agit d'une étude faite corrélativement par EXAFS à LURE, et par diffraction des rayons $X$ et des neutrons [71] sur des monocarbures de titane non stœchiométriques $\mathrm{TiC}_{1-x}$, où $x$ est la concentration des lacunes dans le sous-réseau des atomes de carbone. Elle peut atteindre $50 \%$, et s'accompagne d'un désordre de position qui croît avec $x$ et atténue très fortement l'amplitude des spectres EXAFS (cf. Fig. 13) en fonction de l'énergie. Les pics des transformées de Fourier peuvent être réduits d'un ordre de grandeur dans $\mathrm{TiC}_{0.7}$ par rap-

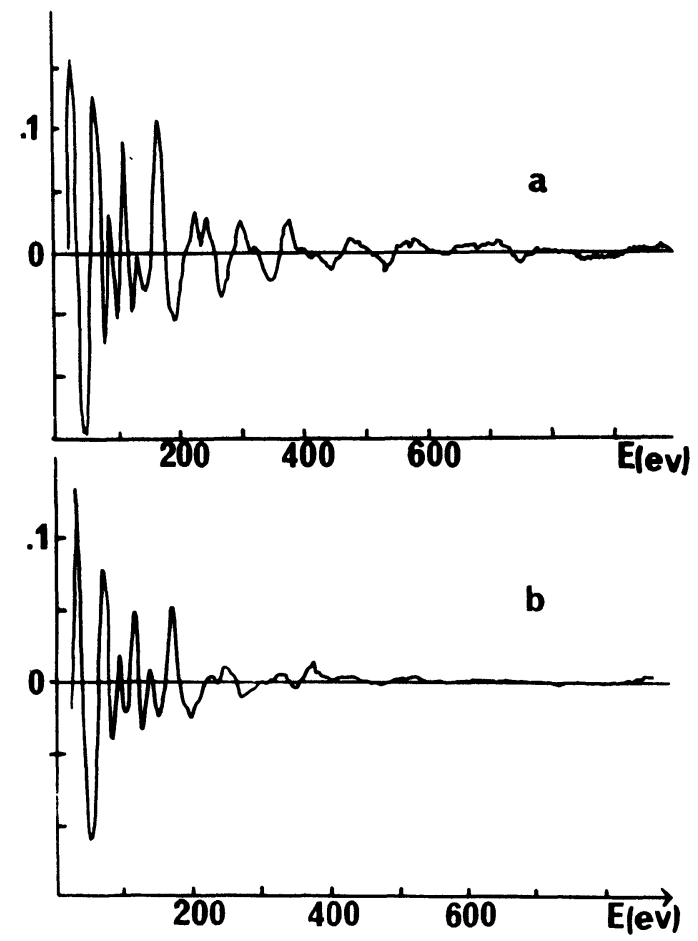

Fig. 13. - Spectre EXAFS de carbures de titane (seuil dı titane) : a) $\mathrm{TiC}$ stæchiométrique, b) $\mathrm{TiC}_{0,7}$.

[EXAFS spectra on the titanium $\mathrm{K}$ edge of : $a$ ) a stoechiometric $\mathrm{TiC}$ sample, $b$ ) a non-stoichiometric $\mathrm{TiC}_{0.7}$ sample.] port à TiC stœchiométrique. Si l'on prend en compte le désordre - ici statique - en utilisant le formalisme de Debye-Waller $\mathrm{e}^{-2 \sigma^{2} k^{2}}$ et la formule classique (3), on obtient des résultats non satisfaisants :

- les distances entre un atome de titane et ses premiers voisins carbone et seconds voisins $\mathrm{Ti}$, déterminées par EXAFS sont inférieures à celles mesurées par diffraction des rayons $\mathrm{X}$ de 2 à $3 \times 10^{-2} \AA$,

- le nombre de deuxièmes voisins $\mathrm{Ti}-\mathrm{Ti}$, qui ne doit pas dépendre de la concentration en lacunes de carbone, décroît de 12 à 10 lorsque l'augmentation de désordre $\Delta \sigma^{2}$ entre $\mathrm{TiC}_{1-x}$ et $\mathrm{TiC}$ croît, et ce pour des valeurs $\Delta \sigma$ inférieur ou de l'ordre de $0,1 \AA$ (Fig. 14).

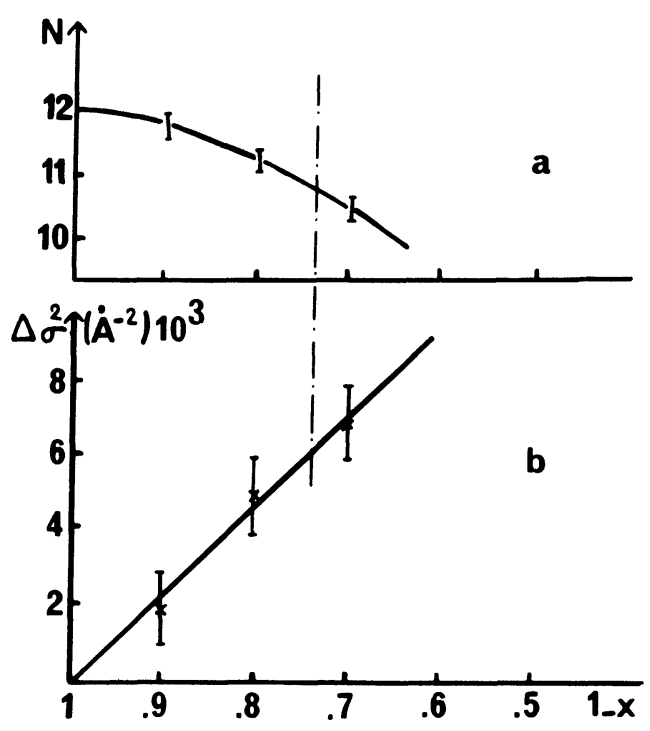

Fig. 14. - Variation en fonction de l'écart à la stœchiométrie entre $\mathrm{TiC}$ et $\mathrm{TiC}_{1-x}: \bar{a}$ ) du nombre de seconds voisins $\mathrm{Ti}$-Ti déterminé par l'EXAFS, $b$ ) de la différence $\Delta \sigma^{2}$ entre les facteurs DebyeWaller $\sigma^{2}$ des spectres EXAFS correspondant à des échantillons $\mathrm{TiC}_{1-x}$ et $\mathrm{TiC}$ stœchiométrique.

[Dependence on the non-stoichiometry $1-x$ of $\mathrm{TiC}_{1-x}$ of : a) the number of Ti second neighbours determined from the EXAFS signal, $b$ ) the static disorder $\Delta \sigma^{2} . \Delta \sigma^{2}$ is the difference between the $\sigma^{2}$ Debye-Waller like damping parameter determined from the EXAFS spectrum of a non stoechiometric $\mathrm{TiC}_{1-x}$ sample and the corresponding value for $\mathrm{TiC}$ stoechiometric.]

Cela montre clairement l'existence d'un effet de fluctuations de distance tant sur l'amplitude que sur la phase du signal $\sigma$, qui n'est pas pris en compte par le formalisme de Debye-Waller. De tels effets limitent la précision que l'on peut atteindre par EXAFS : dès que $\sigma>0,1 \AA$, on ne peut plus prétendre mesurer des distances à moins de $2 \times 10^{-2} \AA$, ni des nombres de coordination à 0,5 unité près. Dans le cas présent, les expériences d'EXAFS, bien que peu utilisables quantitativement, donnent cependant un renseignement qualitatif direct qui n'avait pu être obtenu par diffusion des rayons $\mathrm{X}$ ou des neutrons, à savoir que le désordre dû aux lacunes dans le sous-réseau carbone est à l'échelle atomique, et qu'on n'a pas affaire à des microcristaux désorientés les uns par rapport aux autres. 
Ces effets liés au désordre local ont été étudiés par des simulations sur des distributions modèles de distance par Eisenberger et Brown [36]. Leurs conclusions peuvent se résumer ainsi : pour des systèmes dans lesquels la fonction de distribution des distances a une largeur $\sigma>0,2 \AA$, ou pour lesquels elle est dissymétrique - le critère est $k^{3}\left\langle x^{3}\right\rangle\langle 0,1-$ on ne peut garantir la précision de 1 à $2 \times 10^{-2} \AA$, sauf si on dispose d'un composé modèle ayant la même distribution de distances (ce qui n'est pas toujours le cas), ou si on connaît cette distribution (ce qui est rare). La très grande sensibilité de l'EXAFS au degré de désordre local est due au fait que le transfert de moment $y$ est $2 k$, et pas $k$ comme dans les expériences de diffusion des $\mathrm{X}$ ou des neutrons. Elle tient aussi à ce qu'on n'a pas de théorie simple de l'EXAFS pour des faibles valeurs de $k$ du fait des problèmes théoriques discutés au paragraphe 1.3. En n'analysant les résultats que pour des valeurs de $k$ typiquement supérieures à $5 \AA^{-1}$, soit des transferts de moment de $10 \AA^{-1}$, on se prive donc de la partie de l'information expérimentale qui est la moins affectée par les fluctuations de distance.

Il y a là une limite très réelle d'emploi de l'EXAFS pour l'étude des milieux très désordonnés; encore convient-il de ne pas la surestimer. Dans de nombreux cas d'études des amorphes ou des liquides l'ordre local est suffisamment bien défini pour qu'on puisse tirer des conclusions quantitatives précises, et nous en avons donné plusieurs exemples. Par ailleurs, même si la précision obtenue est plus faible dans le cas de systèmes très désordonnés, il existe toute une gamme d'effets semi-quantitatifs comme par exemple les études d'ordre dans des solutions discutées précédemment, pour lesquels la précision ultime de tout ou partie de l'analyse n'est pas très importante mais où l'essentiel est de montrer directement des changements structuraux que les autres techniques ont du mal à révéler.

\section{Bibliographie}

[1] Dhez, P., Esteva, J. M., Lagarde, P., Fontaine, A., RaOuX, D., Communication Congrès S.F.P. (1979) Toulouse, non publié.

[2] Kossel, W., Z. Phys. 1 (1920) 119.

[3] Kronig, R. de L., Z. Phys. 70 (1931) 317 ; ibid. 75 (1932) 191 ; ibid. 75 (1932) 468.

[4] Petersen, H., Z. Phys. 98 (1936) 569.

[5] Kostarev, A. I., Zh. Eksp. Teor. Fiz. 9 (1939) 267.

[6] Sayers, D. E., Lytle, F. W., Stern, E. A., Adv. X-Ray Anal. 13 (1970) 248.

[7] Sayers, D. E., Lytle, F. W., Stern, E. A., J. Non. Cryst. Solids 8 (1972) 401.

[8] Sayers, D. E., Lytle, F. W., Stern, E. A., Amorphous and Liquid Semiconductors [édité par Stuke J. et Brenig W.] 1 (1974) 403.

[9] Lytle, F. W., Sayers, D. E., Stern, E. A., Phys. Rev. B 11 (1975) 4825

[10] Stern, E. A., Sayers, D. E., Lytle, F. W., Phys. Rev. B 11 (1975) 4836.

[11] Kincaid, B. M., Eisenberger, P., Phys. Rev. Lett. 34 (1975) 1361.

[12] Stern, E. A., Phys. Rev. B 10 (1974) 3027.

[13] LeE, P. A., Pendry, J. B., Phys. Rev. B 11 (1975) 2795.

[14] Ashley, C. A., Doniach, S., Phys. Rev. B 11 (1975) 1279.

[15] Eisenberger, P., Kincaid, B. M., Science 200 (1978) 1441.

[16] Lemonnier, M., Collet, O., Depautex, C., Esteva, J. M., RAOUX, D., Nucl. Instrum. Methods 152 (1978) 109.

[17] Fontaine, A., Lagarde, P., Raoux, D., Esteva, J. M., J. Phys. F 9 (1979) 2143.

[18] Jaklevic, J., Kirby, J. A., Klein, M. P., Robertson, A. S., Brown, G., Eisenberger, P., Solid State Commun. 23 (1977) 679.

[19] Eisenberger, P., Shulman, R. G., Brown, G. S., Ogawa, S., Proc. Natl. Acad. Sci. USA 73 (1976) 491.

[20] Citrin, P. H., Eisenberger, P., Hewit, R., Phys. Rev. Lett. 41 (1978) 309.

[21] Bianconi, A., Bachrach, R. Z., Phys. Rev. Lett. 42 (1979) 104.

[22] Stöhr, J., Denley, D., Perfetti, P., Phys. Rev. B 18 (1978) 4132.

StÖHR, J., J. Vac. Sc. Technol. 16 (1978) 37.

Stöhr, J., Johansson, L. I., Lindau, I., Pianetta, P., Phys. Rev. B 20 (1979) 664.

[23] Parrat, L. G., Rev. Mod. Phys. 31 (1959) 616.
[24] Dehmer, J. L., J. Chem. Phys. 56 (1972) 4496.

[25] Beeman, W. W., Friedman, H., Phys. Rev. 56 (1939) 392.

[26] Cauchois, Y., Mott, N. F., Phil. Mag. 40 (1949) 1260.

[27] Nozières, P., Dominicis, C. T., Phys. Rev. 178 (1969) 1097.

[28] Brown, M., Peierls, R. L., Stern, E. A., Phys. Rev. B 15 (1977) 738.

[29] Heald, S. M., Stern, E. A., Phys. Rev. B 16 (1977) 5549.

[30] Lytle, F. W., Acta Crystallo. 22 (1967) 321.

[31] Calas, G., Levitz, P., Bondot, P., Loupias, G., Petiau, J., Rev. Phys. Appl., ce volume.

[32] Launois, H., Rawiso, M., Wohleben, D., Holland Moritz, E., PotT, R., Communication au colloque des utilisateurs de LURE (1979), non publié.

[33] Noguera, C., Spanjaard, D., Friedel, J., J. Phys. F 9 (1979) 1189.

[34] Pendry, J. B., Low Energy Electron Diffraction (Academic Press) 1974

[35] Lindau, I., SPICER, W. E., J. Electron Spectrosc. and Relat. Phenom. 3 (1974) 409.

[36] Eisenberger, P., Brown, G. S., Solid State Commun. 29 (1979) 481.

[37] Lee, P. A. et Beni, G., Phys. Rev. B 15 (1979) 2862.

[38] Té, B. K., Lee, P. A., J. Am. Chem. Soc. 101 (1979) 2815.

[39] Teo, B. K., Lee, P. A., Simons, A. L., Eisenberger, P. et Kincaid, B. M., J. Am. Chem. Soc. 99 (1977) 3854.

[40] Lee, P. A., Teo, B. K., Simons, A. L., J. Am. Chem. Soc. 99 (1977) 3856

[41] Citrin, P. H., Eisenberger, P., Kincaid, B. M., Phys. Rev. Lett. 36 (1976) 1346

[42] Lagarde, P., Phys. Rev. B 14 (1976) 741.

[43] Fontaine, A., Lagarde, P., Naudon, A., Raoux, D., SpanJAARD, D., Phil. Mag. B $\mathbf{4 0}$ (1979) 17.

[44] Martens, G., Rabe, P., Schwentner, N., Werner, A., Phys. Rev. B 17 (1978) 1481.

[45] Rehr, J. J., Stern, E. A., Martin, R., Davidson, E., Phys. Rev. B 17 (1978) 560

[46] Fontaine, A., Lagarde, P., Raoux, D., Fontana, M. P., Maisano, G., Migliardo, P., Wanderlingh, F., Phys. Rev. Lett. 41 (1978) 504.

[47] Martens, G., Rabe, P., Schwentner, N., Werner, A., Phys. Rev. Lett. 39 (1977) 1411.

[48] Rabe, P., Tolkiehn, G., Werner, A., J. Phys. C 12 (1979) 1173.

[49] Hayes, T. M., J. Non Cryst. Solids 31 (1978) 57. 
[50] Pettifer, R. F., Mc Millan, P. W., Gurman, S. J., The structure of non crystalline materials (ed. P. H. Gaskell), 1977, Londres.

Pettifer, R. F., Mc Millan, P. W., Phil. Mag. 35 (1977) 87.

[51] Sayers, D. E., Stern, E. A., Lytle, F. W., Phys. Rev. Lett. 27 (1971) 1204

[52] Sayers, D. E., Lytle, F. W., Stern, E. A., J. Non Cryst. Solids 8-10 (1972) 401.

[53] Sayers, D. E., Stern, E. A., Lytle, F. W., Phys. Rev. Lett. 35 (1975) 584.

[54] Hunter, S. H., Ph. D. Thesis (Stanford 1977).

[55] Hunter, S. H., Bienenstock, A. B., Hayes, T. M., The structure of non-crystalline materials (ed. P. H. Gaskell) 1977, Londres et Amorphous and liquid semiconductors (ed. W. E. Spear) 1977, Edimbourg.

[56] Hayes, T. M., Allen, J. W., Tauc, J., Giessen, B. C., Hauser, J. J., Phys. Rev. Lett. 40 (1978) 1282.

[57] Knights, J. C., Hayes, T. M., Miknelsen, J. C., Phys. Rev. Lett. 39 (1977) 712.

[58] SADOC, J. F., DIXMIER, J., The structure of non crystalline materials (ed. Gaskell) 1977, Londres, p. 85.

[59] Stern, E. A., Heald, S. M., Bunker, B., Phys. Rev. Lett. 42 (1979) 1372

[60] Crozier, E. D., Lytle, F. W., Sayers, D. E., Stern, E. A., Can. J. Chem. 55 (1977) 1968.

[61] Lytle, F. W., Wong, J., en préparation.
[62] Boyce, J. C., Hayes, T. M., Stutius, W., Miknelsen, J. C., Phys. Rev. Lett. 38 (1977) 1362.

[63] Eisenberger, P., Kincaid, B. M., Chem. Phys. Lett. 36 (1977) 134.

[64] Sandstrom, D. R., Dodgen, H. W., Lytle, F. W., J. Chem. Phys. 67 (1977) 473.

[65] Lagarde, P., Fontaine, A., Raoux, D., Sadoc, A., MIGliardo, P., J. Chem. Phys. 72 (1980) 3061.

[66] Sadoc, A., Fontaine, A., Lagarde, P., Raoux, D., soumis à $J$. Chem. Phys.

[67] Moraweck, B., Clugnet, G., Renouprez, A. J., Surf. Sc. 81 (1979) L 361.

[68] Apai, G., Hamilton, J. F., Stohr, J., Thompson, A., Phys. Rev. Lett. 43 (1979) 165

[69] Sandstrom, D. R., J. Chem. Phys. 71 (1979) 2381.

[70] Michalowicz, A., Girerd, J. J., Goulon, J., Inorg. Chem. 18 (1979) 3004

[71] MoIsy, V., DE Novıon, C. H., communication au Congrès S.F.P. (1979) Toulouse, non publié.

[72] Teo, B. K., Shulman, R. G., Brown, G. S., Meixner, A. E., J. Am. Chem. Soc. 101 (1979) 5624

[73] Via, G. H., Sinfelt, J. H., Lytle, F. W., J. Chem. Phys. 71 (1979) 690.

[74] Brown, G. S., Testardi, L. R., Wernick, J. H., Hallak, A. B., Geballe, T. H., Solid State Commun. 23 (1977) 875. 